\title{
MicroRNA-93 regulates cyclin G2 expression and plays an oncogenic role in laryngeal squamous cell carcinoma
}

\author{
XIYAN XIAO ${ }^{1}$, LIANG ZHOU ${ }^{1}$, PENGYU CAO ${ }^{1}$, HONGLI GONG ${ }^{1}$ and YANPING ZHANG ${ }^{2}$ \\ ${ }^{1}$ Department of Otolaryngology-Head and Neck Surgery and ${ }^{2}$ Central Laboratory, \\ Fudan University Affiliated Eye, Ear, Nose and Throat Hospital, Shanghai 200031, P.R. China
}

Received August 13, 2014; Accepted September 22, 2014

DOI: $10.3892 /$ ijo.2014.2704

\begin{abstract}
RNA93 (miR-93) is expressed in the miR-106b-25 cluster, located in intron 13 of the MCM7 gene. Our previous study found that miR-93 was significantly upregulated in laryngeal squamous cell carcinoma (LSCC), and cyclin G2 (CCNG2) was a potential target of miR-93 in LSCC. However, the possible functions and molecular mechanisms of miR-93 in LSCC remain unknown. In the present study, we show that the level of CCNG2 protein expression was significantly lower in LSCC cancer tissue than normal tissues. The level of CCNG2 was correlated with clinical stages, lymph node metastasis and histological grade. We further show that the expression level of miR-93 was inversely correlated with CCNG2 expression in clinical specimens. Furthermore, gain-of-function assays revealed that miR-93 promoted cell proliferation, decreased apoptosis rates, induced cell cycle arrest and promoted cell migration and invasion, whereas silencing of miR-93 attenuated these carcinogenic processes. In addition, overexpression of miR-93 in Hep-2 cells could reduce the mRNA and protein levels of CCNG2, whereas silencing of miR-93 in Hep-2 cells significantly increased CCNG2 expression. A luciferase assay verified that miR-93 could bind to the 3' untranslated region of CCNG2. Importantly, ectopic expression of CCNG2 in miR-93 cells rescued the effect of miR-93 on LSCC proliferation. Knockdown of CCNG2 promoted cell proliferation resembling that of miR-93 overexpression. These findings demonstrated that miR-93 promotes tumor growth by directly suppressing CCNG2. Taken together, these results suggested that this newly identified miR-93-CCNG2 axis may be involved in LSCC proliferation and progression. Our findings provide novel potential targets for LSCC therapy and prognosis.
\end{abstract}

Correspondence to: Professor Liang Zhou, Department of Otolaryngology-Head and Neck Surgery, Fudan University Affiliated Eye, Ear, Nose and Throat Hospital, No. 83 FenYang Road, Shanghai 200031, P.R. China

E-mail: zhoulent@126.com

Key words: microRNA, miR-93, laryngeal squamous cell carcinoma, cyclin G2, proliferation, cell cycle, apoptosis

\section{Introduction}

Laryngeal squamous cell carcinoma (LSCC) is one of the most common head and neck squamous cell carcinoma (HNSCC) worldwide. Cancer of the larynx accounts for $\sim 1.2 \%$ of new malignancies worldwide every year, with an incidence of $\sim 151,000$ cases per year, $\sim 95 \%$ of these tumors have squamous cell carcinoma as the primary histological type $(1,2)$. It has been reported that this neoplasia is strongly linked to cigarette smoking, alcohol ingestion and human papillomavirus infection (3-5). Treatment options in laryngeal carcinoma comprise surgery, radiotherapy, chemotherapy or a combination therapy. Although treatment options have improved, an epidemiological survey revealed that the survival rate of patients with LSCC has demonstrated a decreasing trend, although the survival rates of patients with other types of cancer have been prolonged (6). Thus, it is necessary and important to look for new diagnostic methods and new treatment targets to control this malignancy.

More than 1,000 miRNAs have been estimated in human genome, only 200-300 miRNAs have been currently identified in humans (7). It has been extensively debated whether miRNAs are just fine-tuning molecules or they act as key gene switches. Recent studies suggest that both hypotheses are probably true, depending on the specific biological context (8). Emerging evidence has demonstrated that miRNAs have diverse functions in regulating multiple stages of cancer such as cell proliferation, initiation, promotion, malignant conversion, progression, angiogenesis, epithelial-to-mesenchymal transition, invasion and metastasis (9). Aberrant expressions of miRNAs have been closely associated with cancer pathogenesis, $>50 \%$ of miRNA-encoding loci located in cancer-associated genomic regions or in fragile sites, some miRNAs have been reported to function as oncogenes or tumor suppressors (10).

miR-93 is a member of the miR-106b-25 cluster, a paralog of the miR-17-92 cluster. miR-106b-25 cluster contains three pre-miRNAs, pre-miR-25, pre-miR-93 and pre-miR-106b, which all reside in the 13th intron of the MCM7 gene. Previous studies indicated that miR-93 can repress the tumor suppressor TP53INP1 in human T-cell leukemia virus 1-transformed human T-cells (11) and FUS-1 in human lung cancer cell lines (12). Ectopic expression of miR-93 decreased expression of NRF2, suggested an oncogenic potential during breast carci- 
nogenesis (13). miR-93 functions as an oncogene by enhancing tumor cell survival, angiogenesis and metastasis by targeting integrin- $\beta 8$ expression (14) and LATS 2 expression (15). miR-93 can also function as a new regulator of PTEN/Akt signaling pathway, and may serve as a potential target for overcoming CDDP resistance in human ovarian cancer (16). Consistently, expression of miR-93 induces mesenchymal-epithelial transition (MET) and downregulates multiple stem cell regulatory genes in 'claudin low' SUM159 cells (17). Considering all the above it would be interesting and meaningful to investigate the role of miR-93 and its family members in LSCC progression.

Cell cycle regulation is the core part in cell proliferation, which has a close relationship with cell carcinogenesis. Cyclin G2 is an unconventional cyclin, located on human chromosome $4 \mathrm{q} 21.1$, function as a ubiquitous inhibitor of cell cycle progression by preventing G1 to S-phase transition. This inhibition is achieved by interacting with protein phosphatase 2A (PP2A), the CCNG2-PP2A complex alters PP2A targeting or its substrate specificity leading to cell cycle arrest. CCNG2 can also stops cell cycle progression by preventing the phosphorylation of cyclin-dependent kinase 2 (CDK2), promoting the formation of unusual nuclear DNA structures suggestive of aberrant mitosis or cytokinesis (18). Phosphoinositide 3-kinase and FoxO transcription factors regulate cyclin G2 expression, thus controling cell cycle progression $(19,20)$. Ectopic expression of cyclin G2 induces microtubule bundling and resistance to depolymerization, inhibition of polymer regrowth from microtubule organizing centers (MTOCs), and a p53-dependent cell cycle arrest (21). These observations suggest that cyclin G2 is a cell cycle modulator that inhibits cellular proliferation. However, the role of CCNG2 is not fully characterized, and the mechanisms of CCNG2 dysregulation in laryngeal cancer are not defined.

In our previous study, we presented comprehensive profiling of miRNAs in LSCC through LCM acquisition of homogeneous samples of cancer cells. Six miRNAs were validated, including upregulation of miR-21, miR-93, miR-205 and miR-708, downregulation of miR-125b and miR-145 (22). We predicted that CCNG2 may be a potential target gene of miR-93. In the present study, we verified that the expression of miR-93 was significantly upregulated in 59 cases of LSCC tissues compared with non-cancerous tissues. Ectopically expressed miR-93 could accelerate growth and invasion of laryngeal cancer cells. Furthermore, we found that CCNG2 is a direct and functional target of miR-93. The expression of CCNG2 is closely correlated with tumor progression and unfavorable outcome.

\section{Materials and methods}

Cell culture. Human embryonic kidney (HEK293) cell line and the human laryngeal squamous carcinoma cell line Hep-2 was purchased from the Cell Bank, Shanghai Institute of Life Science, Chinese Academy of Science (Shanghai, China). The cells were cultured in RPMI-1640 (HyClone, Novato, CA, USA) supplemented with $10 \%$ fetal-bovine serum (FBS; Gibco-BRL, Gaithersburg, MD, USA), penicillin $\mathrm{G}\left(1 \times 10^{5} \mathrm{U} / \mathrm{l}\right)$ and streptomycin $(100 \mathrm{mg} / \mathrm{l})$. These cells were maintained in an incubator at $37^{\circ} \mathrm{C}$ under an atmosphere of $5 \% \mathrm{CO}_{2}$.
Table I. Expression of CCNG2 in LSCC tissue and normal laryngeal tissue.

\begin{tabular}{lrrrrrrrr}
\hline & & \multicolumn{6}{c}{ Expression of CCNG2 protein } \\
\cline { 3 - 8 } Group & Case (n) & - & + & ++ & +++ & $\chi^{2}$ & P-value \\
\hline Cancer tissue & 59 & 38 & 5 & 8 & 8 & 49.015 & $<0.001$ \\
Normal tissue & 59 & 2 & 19 & 17 & 21 & & \\
\hline
\end{tabular}

Clinical specimens. Fifty-nine pairs of laryngeal SCC samples and matched adjacent normal samples were collected from surgical specimens immediately after primary resection of laryngeal carcinoma patients in the Department of Otolaryngology-Head and Neck Surgery, Fudan University Affiliated Eye, Ear, Nose and Throat Hospital, Shanghai, China. The study was approved by the institutional review board and written consent was signed by each patient. None of the patients had received neoadjuvant therapies. The fresh specimens were immediately taken after the surgery, one was fixed in $4 \%$ paraformaldehyde solution, then embedded in paraffin for immunohistochemistry, and the other one was stored in liquid nitrogen for qRT-PCR and western blot assay. Regarding the pathologic evaluation of surgical specimens, the pathologic tumor-node-metastasis classification (TNM), and stages of laryngeal SCC were decided in accordance with the 2002 American Joint Committee on Cancer - Union Internationale Contre le Cancer (AJCC-UICC) TNM system. The LSCC clinical information was collected from patient records, and the details are listed in Tables I and II with the correlation of CCNG2 expression.

Immunohistochemistry. Paraffin-embedded tissues were cut into 4- $\mu \mathrm{m}$ thick sections and analyzed with immunohistochemical staining. CCNG2 and Ki-67 expression was determined based on the percentage of positive cells, combined with the staining intensity. The proportion of positive cells was divided into four levels: 0 point, $\leq 5 \%$ of positive cells; 1 point, $5 \%$ $\sim 25 \%$; 2 points, $25-50 \%$, and 3 points, $>50 \%$ of positive cells. The intensity of staining was classified as: 0 point, no staining; 1 point, weak staining (light yellow); 2 points, moderate staining (brown); and 3 points, strong staining (yellowish brown). The final scores of CCNG2 and $\mathrm{Ki}-67$ expression was the product of the $\mathrm{CCNG} 2$ and $\mathrm{Ki}-67$ expression proportion and intensity, graded as 0 for negative, + for $1-3$ points, ++ for 4-6 points, and +++ for 7-9 points.

In vivo assays for tumor formation. For the in vivo tumor formation assays, $3 \times 10^{6}$ Hep- 2 cells infected with the Lenti-miR-93 or mock vector were suspended in $200 \mu$ l PBS and subcutaneously injected into the right flank of each nude mouse (female BALB/c-nu/nu, four-week-old, 6 per group). Tumor volumes were measured using vernier calipers every 3 days as soon as the tumors were measurable, and the tumor volumes were calculated: $\mathrm{V}\left(\mathrm{cm}^{3}\right)=$ width ${ }^{2}\left(\mathrm{~cm}^{2}\right)$ x length $(\mathrm{cm}) / 2$. On day 32 , the mice were sacrificed. The mice were manipulated and housed according to protocols approved by the Shanghai Medical Experimental Animal Care Commission. 
Table II. The relationship between the expression of cyclin G2 and clinicopathological parameters.

\begin{tabular}{|c|c|c|c|c|c|}
\hline & \multirow[b]{2}{*}{ Case (n) } & \multicolumn{4}{|c|}{ Expression of CCNG2 protein } \\
\hline & & Negative & Positive $(+\sim+++)$ & $\chi^{2}$ & P-value \\
\hline \multicolumn{6}{|l|}{ Age at surgery, (years) } \\
\hline$\leq 65$ & 48 & 17 & 31 & 0.161 & 0.688 \\
\hline$>65$ & 12 & 5 & 7 & & \\
\hline \multicolumn{6}{|l|}{ Gender } \\
\hline Male & 54 & 21 & 33 & 0.002 & 0.961 \\
\hline Female & 5 & 2 & 3 & & \\
\hline \multicolumn{6}{|l|}{ Clinical stage } \\
\hline I-II & 10 & 3 & 7 & 5.51 & 0.019 \\
\hline III-IV & 49 & 34 & 15 & & \\
\hline \multicolumn{6}{|l|}{ Site } \\
\hline Supraglottic & 24 & 6 & 18 & 0.287 & 0.592 \\
\hline Glottic and infraglottic & 35 & 11 & 24 & & \\
\hline \multicolumn{6}{|l|}{ Lymph node metastasis } \\
\hline Negative & 25 & 12 & 13 & 9.451 & 0.002 \\
\hline Positive & 34 & 29 & 5 & & \\
\hline \multicolumn{6}{|l|}{ Histology grade } \\
\hline SCC I-II & 37 & 25 & 12 & 8.984 & 0.003 \\
\hline SCC III & 22 & 6 & 16 & & \\
\hline
\end{tabular}

Lentivirus production and infection. The pri-miR-93 sequence was amplified from normal genomic DNA and cloned into the pGMLV-PE1 microRNA lentiviral vector to generate pGMLVPE1-miR-93. The primer sequences are: miR-93-F (XhoI): CCGCTCGAGAACCTTCACTGAGAGGGTGGTT and miR-93-R (BamHI): CGGGATCCGAGGGAGACCAGA CCCTTTTG. The amplified sequences were inserted into the $\mathrm{XhoI}$ and BamHI restriction sites of the multiple cloning site of expression vector pGMLV-PE1 and verified by sequencing. The shuttle vector was co-transfected with virapower packaging mix, containing packaging plasmid pMDL-gp-RRE, pRSV-Rev, pCMV-VSVG and so on, into 293T cells to produce the lentivirus, using the HG transgene reagent (Genomeditech Co., Shanghai, China). Virus-containing media was collected, filtered and added to target Hep-2 cells for infection in the presence of polybrene (Sigma, St. Louis, MO, USA).

Construction of the pcDNA3.1-CCNG2 and pcDNA3.1-NC expression vectors. The coding region of the CCNG2 mRNA was amplified by PCR from human genomic DNA. The amplified sequences were inserted into the EcoRI and BamHI restriction sites of the multiple cloning site of expression vector pcDNA3.1 and verified by sequencing. The primers used were: human CCNG2-F (EcoRI): CCGGAATTCGCCA CCATGAAGGATTTGGGGGCAG, human CCNG2-R (BamHI): CGGGATCCCTAAGATGGAAAGCACAGTGT TTGT. Empty plasmid was used as negative control. Cells were transfected with the specified expression vector pcDNA3.1 using Lipofectamine 2000. After 48 h, antibioticG418 at $800 \mathrm{ng} / \mathrm{ml}$ was added to select cells with stably integrated expression constructs. Stable cell lines were established within 3-4 weeks.

Cell proliferation and colony formation assays. The cell proliferation assay was measured using the Cell Counting Kit-8 (CCK-8; Dojindo Laboratories, Kumamoto, Japan). Approximately $2 \times 10^{3}$ cells were seeded in each well of a 96-well plate, and $10 \mu \mathrm{l}$ of CCK-8 was added to $90 \mu \mathrm{l}$ of the culture medium at the indicated time. The cells were subsequently incubated at $37^{\circ} \mathrm{C}$ for $2 \mathrm{~h}$, and the ultraviolet absorbance at $450 \mathrm{~nm}$ was measured using a BioTek synergy H1 hybrid reader. For the colony formation assay, 200 cells were plated into each well of a 6 -well plate and incubated at $37^{\circ} \mathrm{C}$ for two weeks, the culture medium was changed once every 3 days. The colonies were fixed and stained in a dye solution containing $1 \%$ crystal violet, and the number of colonies was counted. Colonies were counted using ImageJ software and differences were assessed by two-tailed t-test. These assays were replicated three times.

Migration and invasion assays. A 24-well plate containing $8-\mu \mathrm{m}$-pore size chamber inserts (Corning Incorp.) was used to evaluate the migration and invasion of tumor cells. For the invasion assay, the membrane was coated with Matrigel to form a matrix barrier, and $2 \times 10^{5}$ cells were placed in the upper chamber. For the migration assay, only $5 \times 10^{4}$ cells were seeded in the upper chamber. Cells in $200 \mu 1$ serum-free 1640 medium were gently loaded onto each filter insert (upper chamber) and in each lower chamber, $600 \mathrm{ml}$ of 1640 medium with $10 \%$ FBS was added, then incubated at $37^{\circ} \mathrm{C}$ for 18 or 
$24 \mathrm{~h}$, respectively for migration assay and invasion assay. After that, the filter inserts were removed from the chambers, cells that migrated or invaded were fixed and stained with haematoxylin for $20 \mathrm{~min}$. The number of cells that had migrated or invaded was counted and photographed using a Nikon Ti-E inverted microscope.

Cell cycle and cell apoptosis analysis. Cell Cycle Detection kit was purchased from KeyGen Biotech Co., Ltd. (Nanjing, China). Approximately $1 \times 10^{6}$ cells were collected and fixed with ice-cold $70 \%$ ethanol in PBS at $-20^{\circ} \mathrm{C}$ overnight. Cells were treated with RNase A for $30 \mathrm{~min}$ and then labeled for 15 min with PI and immediately analyzed by flow cytometry. The test was performed in triplicate. The data were evaluated using ModFit software program and presented as means \pm SD $(n=3)$.

The cell apoptosis assay was performed using flow cytometry and was detected with the Annexin V-FITC/PI apoptosis detection kit (KeyGen Biotech) or Annexin V-APC/7-AAD apoptosis detection kit (KeyGen Biotech). Cells were cultured and treated with the agents indicated in the figure legends according to the manufacturer's instructions. Cells were resuspended in $400 \mu \mathrm{l}$ Annexin $\mathrm{V}$ binding buffer and subsequently incubated with $5 \mu \mathrm{l}$ Annexin V-FITC or Annexin V-APC for $15 \mathrm{~min}$ in room temperature; then, $10 \mu \mathrm{l}$ of PI or 7-AAD was added. Experimental data were analyzed using FlowJo software (version 7.6.1) and presented as means $\pm \operatorname{SD}(n=3)$.

Real-time quantitative PCR. For qRT-PCR of miRNAs, lowmolecular-weight RNA ( $<200 \mathrm{nt})$ was separated from the total RNA using the mirVana miRNA isolation kit (Ambion, Foster City, CA, USA) according to the manufacturer's protocol. Normalization was performed using the signal of U6 snRNA. cDNA synthesis was performed with $100 \mathrm{ng}$ total RNA using a Hairpin-it ${ }^{\mathrm{TM}}$ MicroRNAs Quantitation PCR kit (GenePharma, Co., Ltd., Shanghai, China) according to the manufacturer's protocol. The sequences of primers specific for individual miR-93 are as follows: forward primer, CGTTATATCCC AAAGTGCTGTTC and reverse primer, TATGGTTGTTCT CGTCTCCTTCTC. Thermal cycling and fluorescent monitoring were performed on an $\mathrm{ABI} \mathrm{ViiA}^{\mathrm{TM}} 7$ Sequence Detection System. Relative expression (RE) of the sample gene was calculated using the formula $R E=2^{-\Delta \Delta C T}$.

For qRT-PCR of CCNG2 and MMP-9, total RNA was extracted from clinical specimens or cultured cells using TRIzol reagent (Invitrogen). The quantity was measured on a BioTek synergy $\mathrm{H} 1$ hybrid reader, and the integrity of the RNA was checked on a $1 \%$ agarose gel. cDNA was synthesized with the PrimeScript ${ }^{\mathrm{TM}}$ RT Master Mix (Takara Bio, Shiga, Japan) using $500 \mathrm{ng}$ of RNA. The real-time PCR analyses were performed using SYBR ${ }^{\circledR}$ Premix Ex Taq ${ }^{\mathrm{TM}}$ (Takara). The levels of CCNG2 transcript were measured by forward primer, 5'-CAGGATTGAGAAATGCCAAAGT-3' and reverse primer, 5'-TGACAGCCAGGACAAAAGTT-3'. The levels for MMP-9 were measured by forward primer, 5'-GCCACTTCCCCTTC ATCTTC-3' and reverse primer, 5'-GGTCGTCGGTGTCG TAGTTG-3'. $\beta$-actin was used as internal controls and amplified with forward primer, 5'-AGCGAGCATCCCCC AAAGTT-3' and reverse primer, 5'-GGGCACGAAGGCTC ATCATT-3'.
Western blots. Cell lysates were prepared using RIPA buffer (Beyotime Institute of Biotechnology, Shanghai, China). Protein concentration was determined using the BCA assay (Beyotime Institute of Biotechnology). For electrophoresis, equal amounts of cell lysate protein $(30 \mu \mathrm{g})$ were resolved by SDS-PAGE and transferred to polyvinylidene difluoride (PVDF) membrane (Millipore, Billerica, MA, USA). Blots were probed at $4^{\circ} \mathrm{C}$ overnight with primary antibodies in 5\% milk/TBST. Membranes were blocked and probed with the following antibodies: mouse anti-CCNG2 (Abcam BioSciences), mouse anti-GADPH (WeiAo Biotechnology, Shanghai, China). Bound antibodies were detected with secondary HRP-conjugated antibodies and visualized by enhanced chemiluminescent substrate (WeiAo Biotechnology). Western blot data were quantified by normalizing the signal intensity of each sample to that of GADPH using ImageJ software.

Luciferase reporter assay. We used a luciferase reporter vector pLUC to generate luciferase reporter constructs. There are two potential binding sites for miR-93 in the CCNG2 3'UTR. These two sites are close to each other, which made the analysis of each site individually difficult. The segment of the wild-type 3'UTR of CCNG2 (CCNG2-3'UTR) containing the predicted miR-93 target site was cloned from human genomic DNA. The mutant construct (CCNG2-3'UTRmutant), with the part of the target sites corresponding to the seed sequence of miR-93, CCNG2 3'UTR-186 (5'-CAUUA ACAGUACUUUA-3') replaced by (5'-CAUUAACAG UACUUUA-3'), CCNG2 3'UTR-222 (5'-GCACUUU-3') replaced by (5'-CGUGAAA-3'). The CCNG2 fragment was cloned with two primers CCNG2 3'UTR-F XhoI: CACAACT CGAGTCCTGGTTTAGCCCCCATCT, CCNG2 3'UTR-R BamHI: AAGGATCCATGGCTGTATCACCACACAGA. The PCR product was digested with XhoI and BamHI, followed by insertion into a XhoI- and BamHI-opened vector. To generate a mutant containing a mutation in the second site of the 3'UTR (leaving the other site active), the primers were used as follows: CCNG2 3'UTR-MF1: CATTGCGTGAAA ATTTTTCTCGTAGATCTTTAGCTAC, CCNG2 3'UTRMR1: GAAAAATTTTCACGCAATGTCTAAAGTACTG TTAATGG. To generate a mutant containing mutations in both sites, the primers used were: CCNG2 3'UTR-MF2: AAACGTGTTTGTCGTGAAAAGACATTGCGTGAAA ATT, CCNG2 3'UTR-MR2: ATGTCTTTTCACGACAAAC ACGTTTAGAGGCACTACC. For luciferase assays, HEK 293T cells were seeded in 96-well plates at a density of 5,000 cells/well. After $24 \mathrm{~h}$, the cells were transiently transfected with $5 \mathrm{ng}$ of pRL-CMV Renilla luciferase reporter, $30 \mathrm{ng}$ of either CCNG2-3'UTR or CCNG2-3'UTR-mutant and 5 pmol of miRNA mimics. After $48 \mathrm{~h}$, the luciferase activity was measured using the dual-luciferase reporter assay system (Promega, Madison, WI, USA), detected by Tecan Infinite F200/M200 microplate reader. Firefly luciferase activity was normalized to Renilla luciferase activity to evaluate the effect of miR-93 mimics.

Transfection of Hep-2 cells with miRNA mimics, inhibitor or siRNAs. All miRNAs mimics and inhibitors were synthesized by GenePharma. The siRNAs specific for CCNG2 were synthesized by Biotend (Shanghai, China). The oligonucle- 

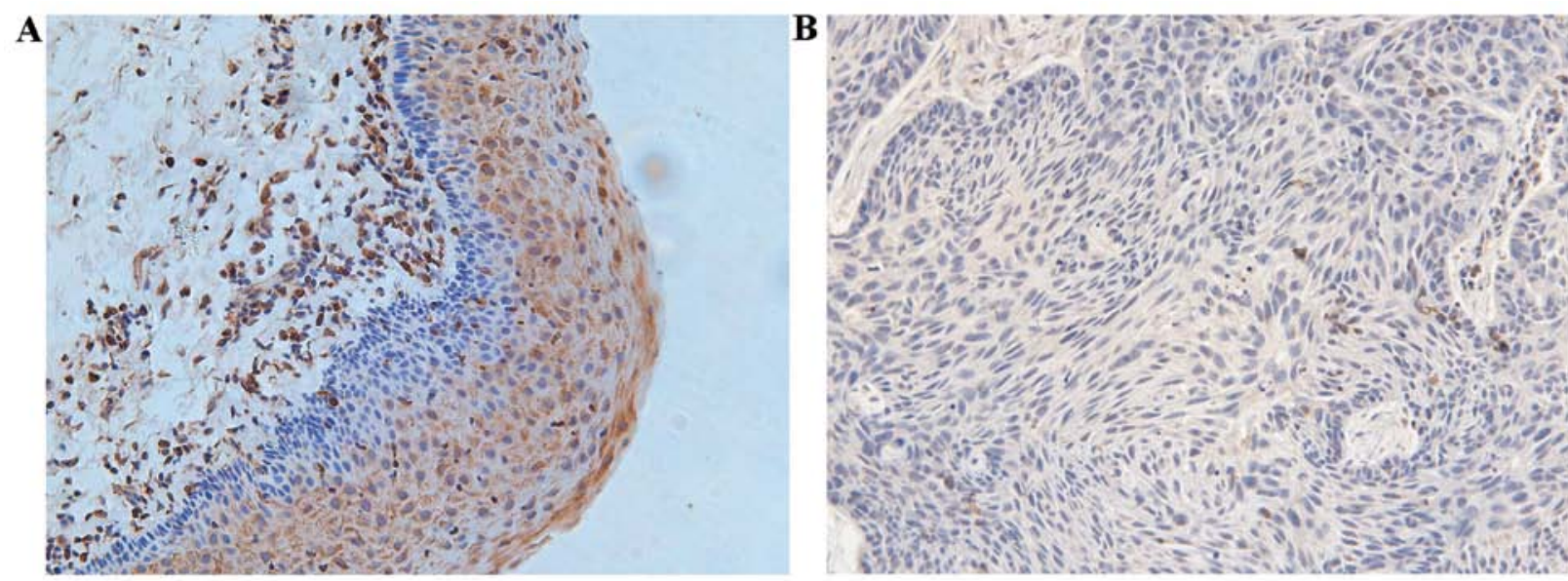

C

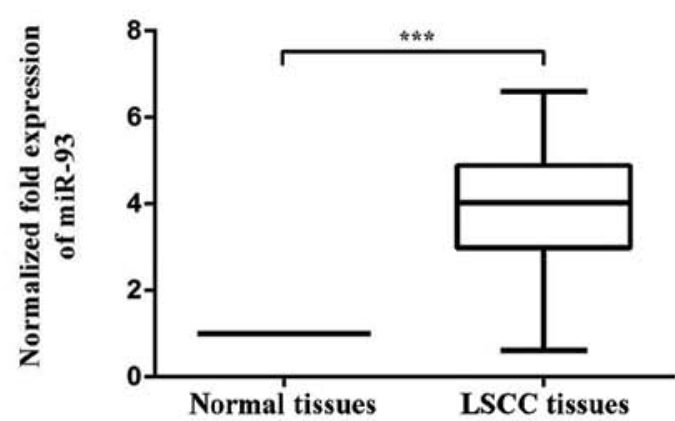

D

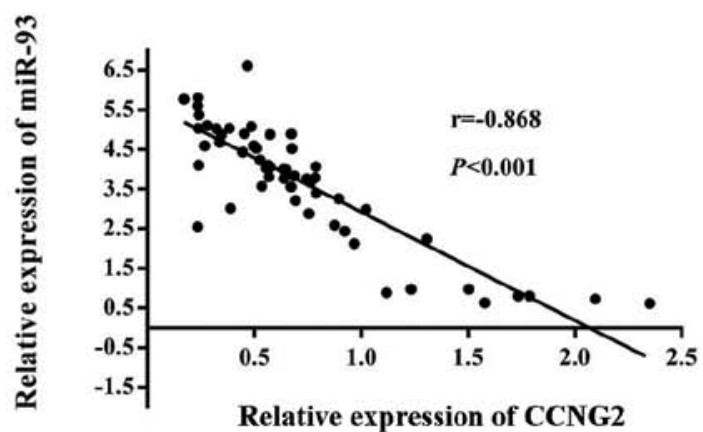

Figure 1. CCNG2 is more often reduced in LSCC than adjacent non-neoplastic tissues and its expression is inversely correlated with miR-93 in LSCC. (A) Adjacent non-neoplastic tissue: strong positive staining is visible in the cytoplasm (magnification, $\mathrm{x} 200$ ). (B) LSCC: the staining is not evident in the cytoplasm (magnification, x200). (C) The relative expression of mature miR-93 in 59 pairs of LSCC tissues and their corresponding non-cancerous tissues using the $2^{-\triangle \Delta C T}$ method. (D) The correlation between CCNG2 mRNA expression and mature miR-93 was analyzed in the same LSCC samples by linear regression. The expression data were normalized to $\beta$-actin and U6 snRNA, respectively.

otide transfection was performed using Lipofectamine 2000 (Invitrogen, Carlsbad, CA, USA).

miRNA mimis $(50 \mathrm{nmol})$, inhibitor $(100 \mathrm{nmol})$ or siRNAs transfection was performed using Lipofectamine 2000 following the manufacturer's protocol. In brief, $4 \times 10^{4}$ cells in $1.5 \mathrm{ml}$ of RPMI-1640 (10\% FBS) were plated in each of five different $35-\mathrm{mm}$ tissue culture dishes and were incubated overnight at $37^{\circ} \mathrm{C}$ and $5 \% \mathrm{CO}_{2}$ atmosphere. For each dish, $5 \mu \mathrm{l}$ mimics or $10 \mu \mathrm{l}$ inhibitor or $10 \mu \mathrm{l}$ siRNA was added into $250 \mu \mathrm{l}$ of serum-free medium and mixed with $5 \mu 1$ of Lipofectamine 2000. The mixture was added to cells and incubated for $6 \mathrm{~h}$ before replacing the medium with complete medium.

Three siRNAs that were synthesized to target CCNG2 expression were used in this experiment: 5'-3' Si-1: sense, GGC UGCUAGAAUAGUUGAAdTdT and antisense, UUCAAC UAUUCUAGCAGCCdTdT; Si-2: sense, CUCCACAACAG CUACUAUA and antisense, UAUAGUAGCUGUUGUGG AG; Si-3: sense, CCCGGAGAAUGAUAACACU and antisense, AGUGUUAUCAUUCUCCGGG; Si-NC: sense, uucuc cgaacgugucacguTT and antisense, acgugacacguucggagaaTT.

Statistical analysis. All statistical analyses were performed using SPSS 20.0 software. For the clinicopathological features, P-values were calculated using the $\chi^{2}$ test. The results (mean values $\pm \mathrm{SD}$ ) of all the experiments were subjected to statistical analysis by the Student's t-test (two-tailed, with $\mathrm{P}<0.05$ considered significant). Error bars depict SD. Asterisks were used to represent statistical significance of P-values in the figures, e.g. ${ }^{*} \mathrm{P}<0.05,{ }^{* *} \mathrm{P}<0.01,{ }^{* * *} \mathrm{P}<0.001$.

\section{Results}

Differential expression of CCNG2 in LSCC and adjacent nonneoplastic tissues. If miR-93 actually regulates the expression of CCNG2 in LSCC, then the expression of these two factors should be inversely correlated in LSCC. Therefore, we evaluated the expression of CCNG2 mRNA in corresponding the previous 59 cases of LSCC and the adjacent non-cancerous laryngeal tissues. CCNG2 mRNA was downregulated in LSCC tissues compared with their respective non-cancerous laryngeal tissues. For the immunohistochemical staining, staining was negative or weak in LSCC tissues, while in normal laryngeal tissues, CCNG2 staining ranged from light yellow to brown (Fig. 1A and B). Statistically, CCNG2 was expressed in $35.6 \%$ (21/59) of laryngeal cancer tissues, which was lower than $96.6(57 / 59)$ in normal tissues. The difference was statistically significant $(\mathrm{P}<0.001)$ (Table I). The expression of CCNG2 was correlated with clinical stages, lymph node metastasis and pathological differentiation $(\mathrm{P}<0.05)$, regardless of age, gender and tumor site $(\mathrm{P}>0.05)$ (Table II).

CCNG2 mRNA expression in LSCC was negatively correlated with miR-93 expression. To validate the expression of miR-93 in LSCC, we detected mature miR-93 in 59 pairs of LSCC and 
A

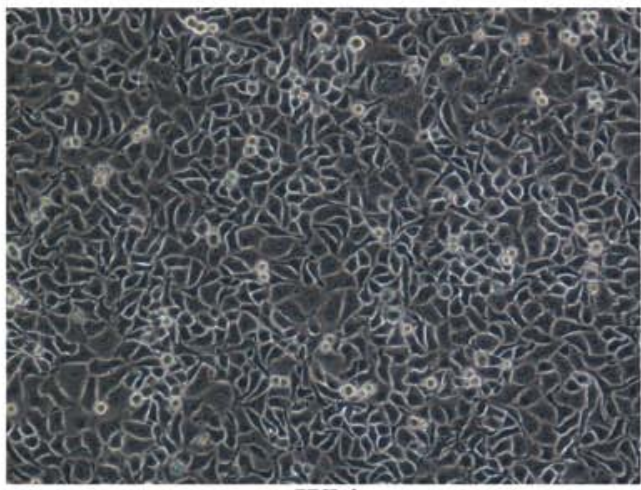

B

White

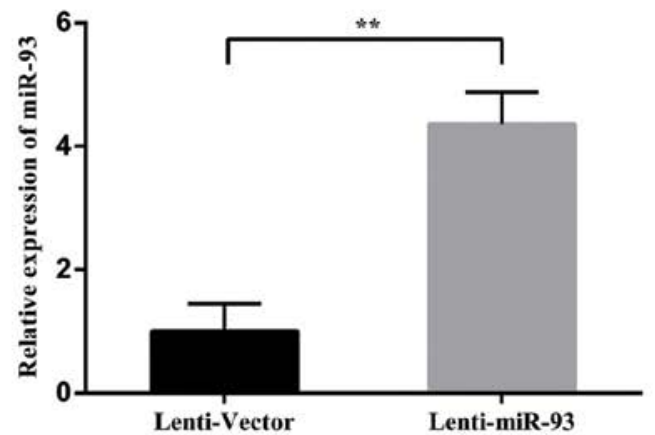

C

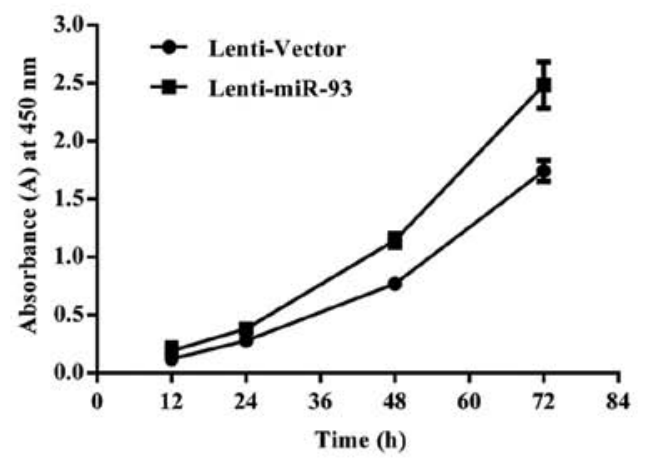

D

Lenti-Vector

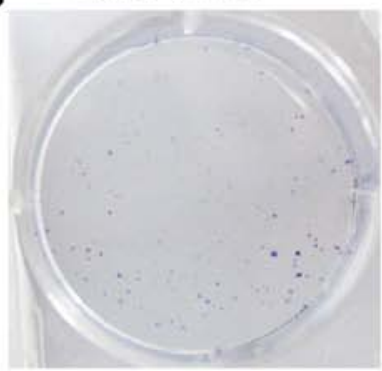

Lenti-miR-93

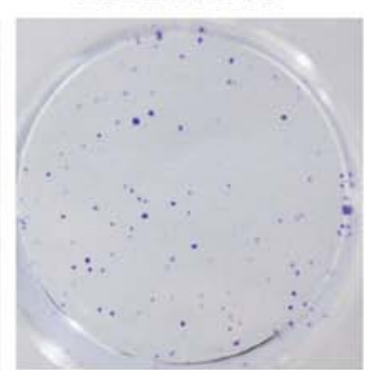

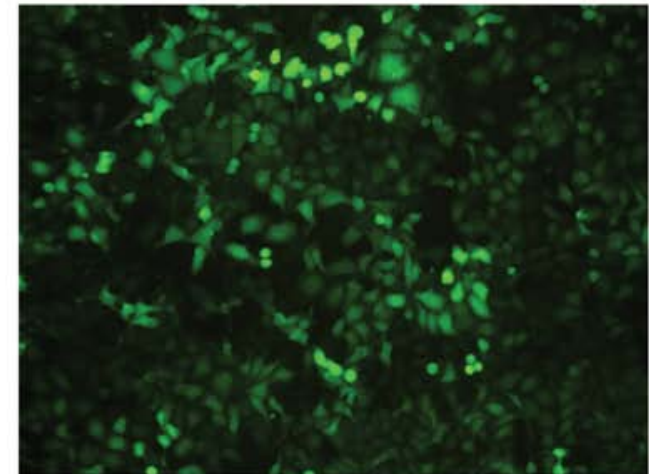

Green
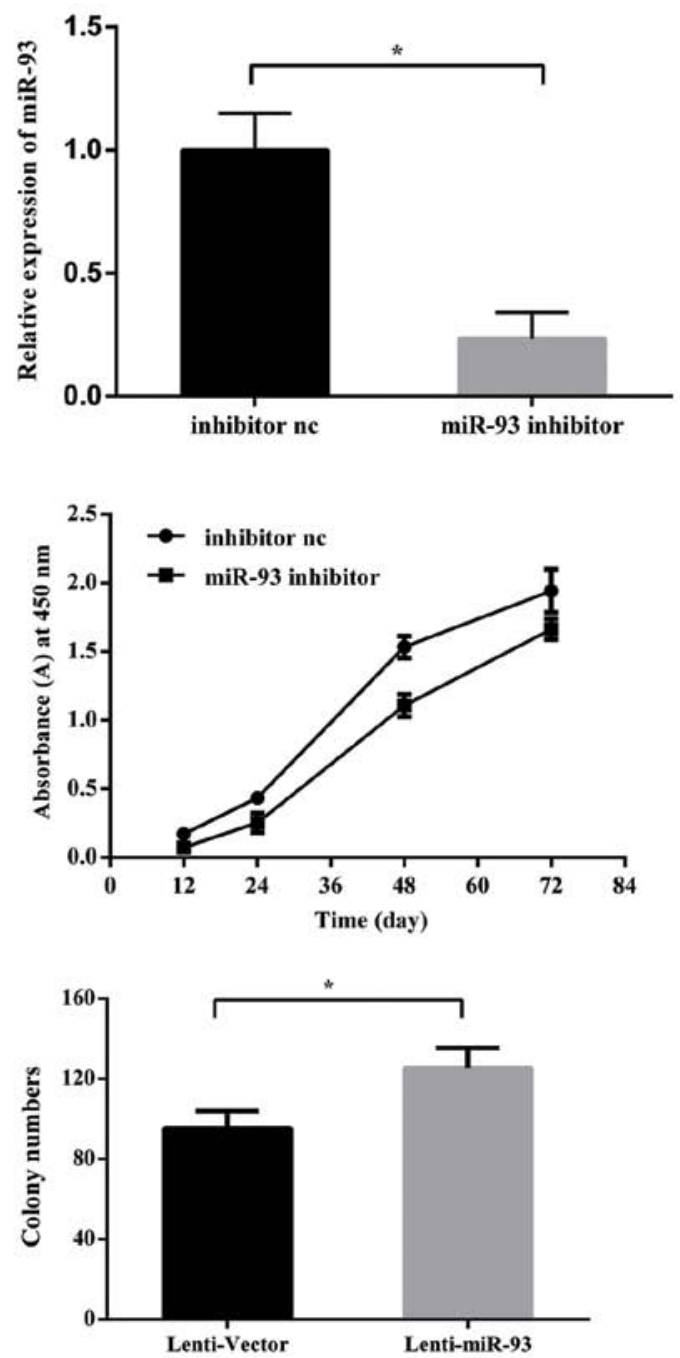

Figure 2. miR-93 promotes cancer cell proliferation in vitro. (A) Establishment of stable cell lines highly expressing miR-93, effectiveness of infection of Hep-2 cells with Lenti-miR-93 (magnification, x200). White: white light used as the excitation light; green: blue light as the excitation light. (B) The relative expression level of mature miR-93 in Hep-2 cells infected with lentivirus expressing miR-93 or mock vector, and Hep-2 cells transfected with miR-93 inhibitor or negative control. Data are normalized by U6 snRNA. (C) Cell proliferation assays for upregulation and downregulation of miR-93 were conducted using the Cell Counting Kit-8 (CCK-8) assay. (D) The colony formation assays for Hep-2 cells infected with lentivirus expressing miR-93 or the mock control.

matched non-cancerous laryngeal tissues by real-time PCR. The results indicated that miR-93 expression was upregulated in $86.44 \%$ of LSCC tissues compared with the non-cancerous laryngeal tissues, at 3.73-fold higher mean level (Fig. 1C).

Moreover, the downregulation of CCNG2 was inversely correlated with the upregulation of miR-93 in these LSCC samples. The Pearson correlation was recorded between CCNG2 and miR-93 expression ( $\mathrm{r}=-0.868, \mathrm{P}<0.001)$ (Fig. 1D). These data suggest that CCNG2 mRNA expression is negatively correlated with miR-93 expression in LSCC tissues and miR-93 may have an important role in cell cycle and proliferation. 
A

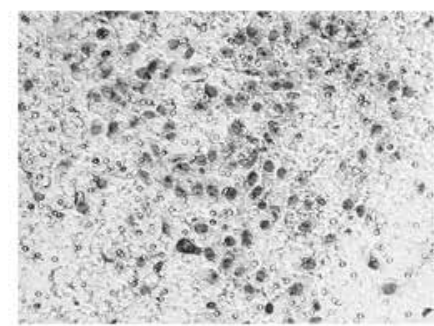

Lenti-vector

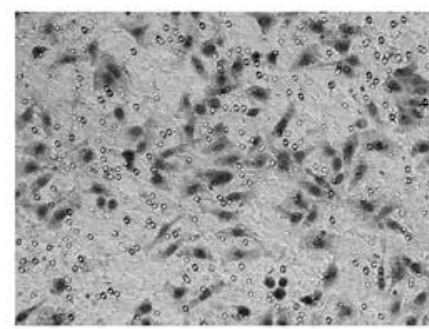

NC inhibitor
Migration assay

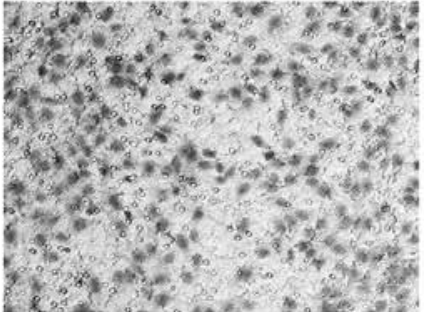

Lenti-miR-93

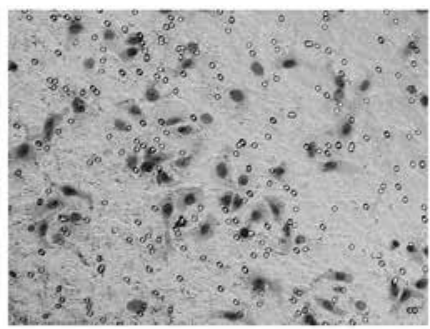

miR-93 inhibitor
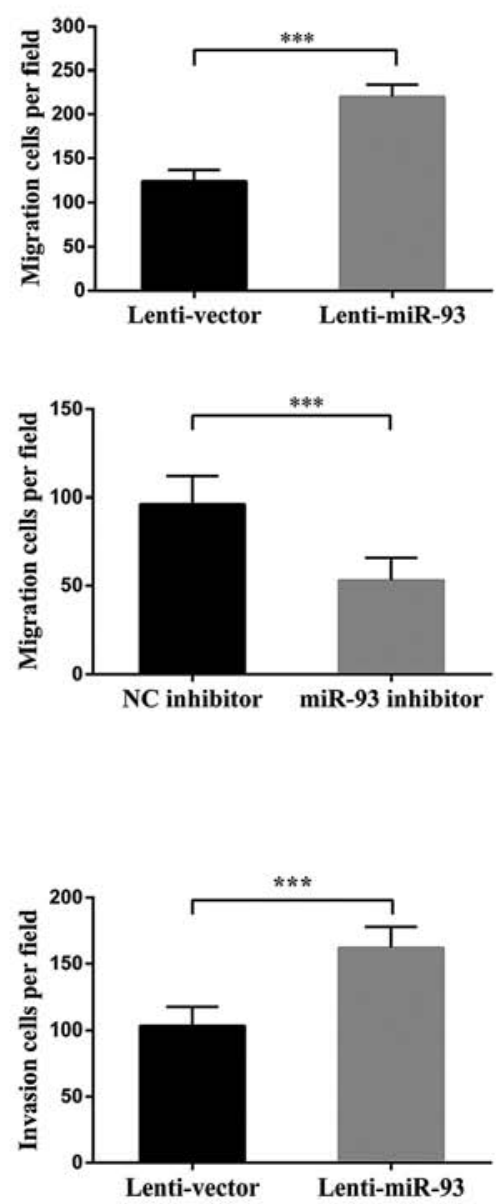
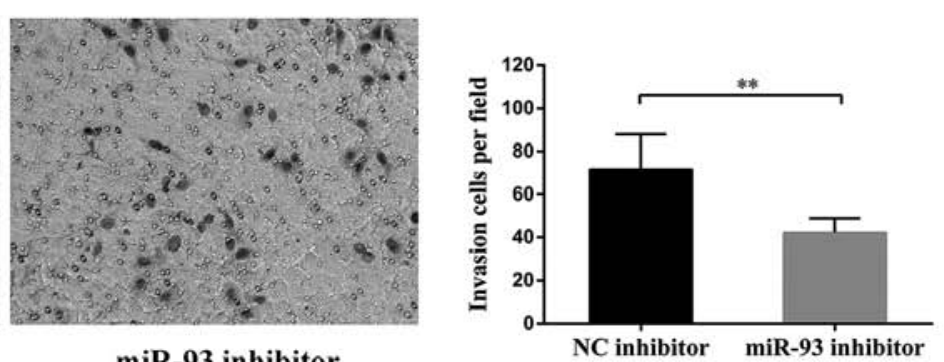

Lenti-miR-93

miR-93 inhibitor

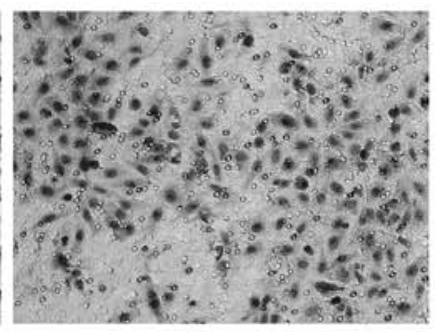

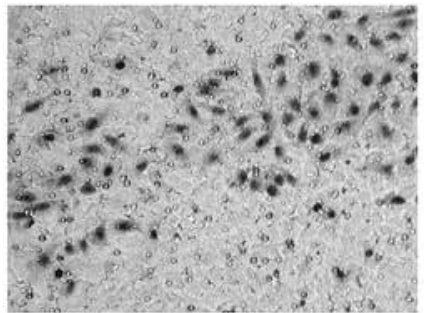

NC inhibitor

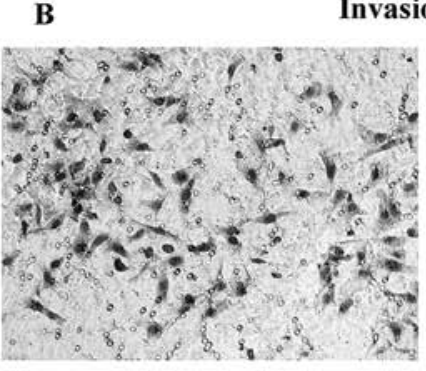

Lenti-vector

Figure 3. miR-93 enhances LSCC cell invasion and metastasis in vitro. (A) Transwell migration assays of Hep-2 cells were performed after infection with miR93-expressing or control lentivirus, and additional assays were performed after Hep-2 cell transfection with the miR-93 inhibitor or the negative control. (B) Transwell invasion assays of Hep-2 cells were performed after infection with miR-93-expressing or control lentivirus, and additional assays were performed after Hep-2 cells transfection with the miR-93 inhibitor or the negative control.

miR-93 promotes the proliferation and colony formation in vitro. To examine the biological function of miR-93 in the development of LSCC, a lentivirus vector expressing miR-93 was constructed and used to infect Hep-2 cells to establish stable cell lines, denoted as Lenti-miR-93 (Fig. 2A), the negative control as Lenti-Vector after lentivirus transduction. The silencing of miR-93 was via the tansfection of Hep-2 cells with miR-93 inhibitor and the inhibitor negative control. The relative expression levels of mature miR-93 were detected using qRT-PCR (Fig. 2B). Cell proliferation assays revealed that the ectopic expression of miR-93 in these stable cancer cells resulted in a significant increase in cell proliferation. In contrast, the silencing of miR-93 reduced the cell proliferation rates compared with the negative control (Fig. 2C). To further examine the effect of miR-93 on the selfrenewing capacity in vitro, Lenti-miR-93 cells stably expressing miR-93 or the vector control were plated at a density of 200 cells/well in 6-well cell cultrue cluster, Lenti-miR-93 cells generated a greater number of tumor spheres than the vector control (95.33 \pm 8.5 and $125.30 \pm 10.0$, respectively) (Fig. 2D). miR-93 overexpression in laryngeal cancer cells significantly promotes long-term cell growth as measured by colony formation assay.

miR-93 promotess cancer cell invasion and metastasis in vitro. miR-93 was recently reported to promote the metastasis of breast cancer cells in vitro (15), thus, prompting us to 
A

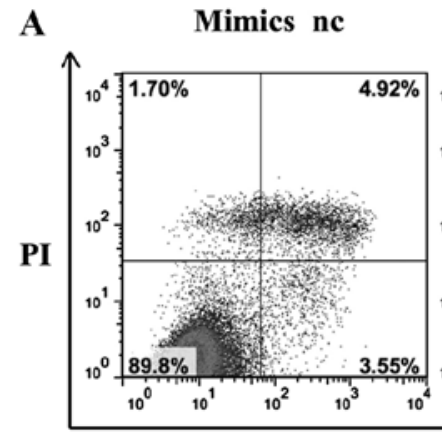

miR-93 mimics

Inhibitor ne

miR-93 inhibitor
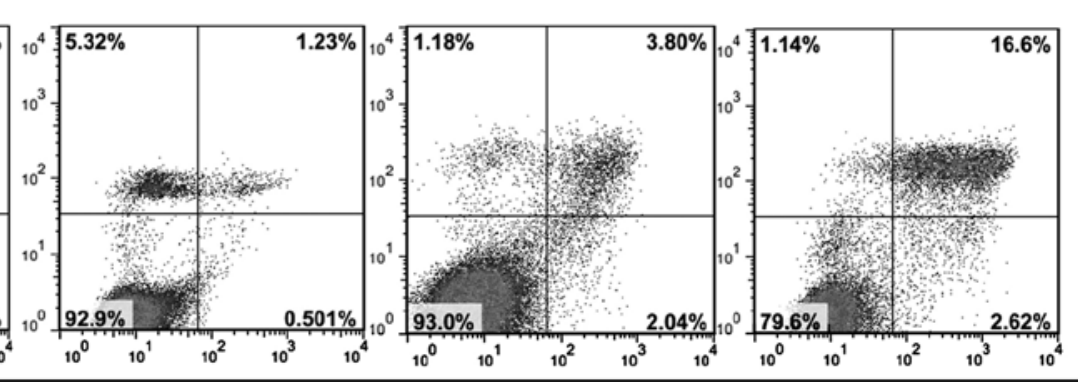

Annexin V-FITC
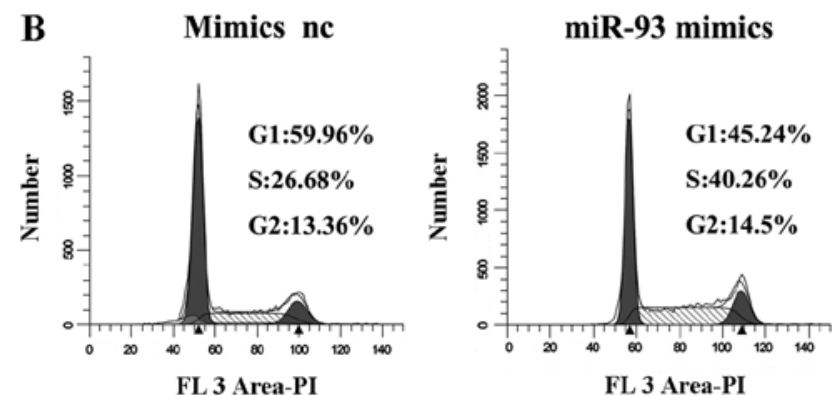

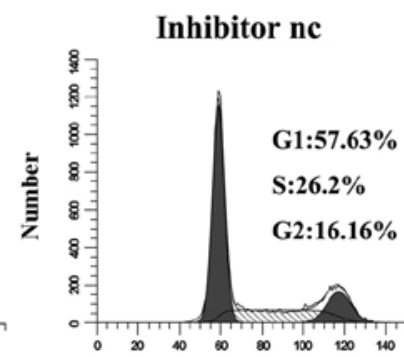

FL 3 Area-PI

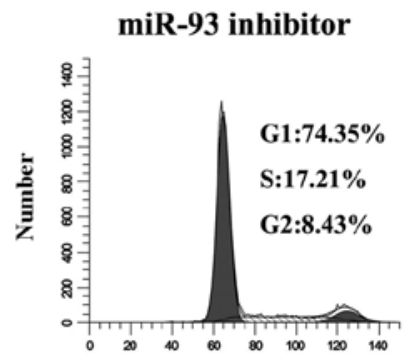

FL 3 Area-PI

Figure 4. miR-93 inhibits Hep-2 cell apoptosis in vitro and promotes the cell cycle of Hep-2 cells. (A) Cell apoptosis rates of Hep-2 cells transfected with miR-93 mimics were significantly decreased compared with the mimics nc group. Cell apoptosis rates of Hep-2 cells transfected with miR-93 inhibitor were significantly increased compared with the inhibitor nc group. (B) Overexpression of miR-93 induced cell cycle arrested at the S phase in Hep-2 cells, while downregulation of miR-93 induces cell cycle arrested at the G1 phase in Hep-2 cells.

A
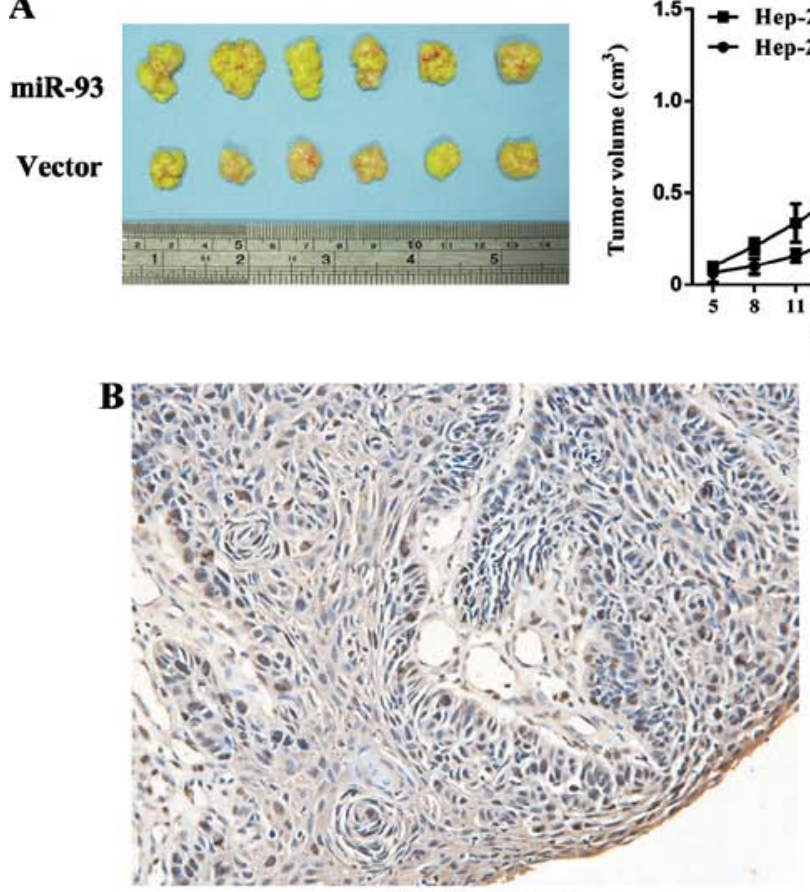

Hep-2 vector
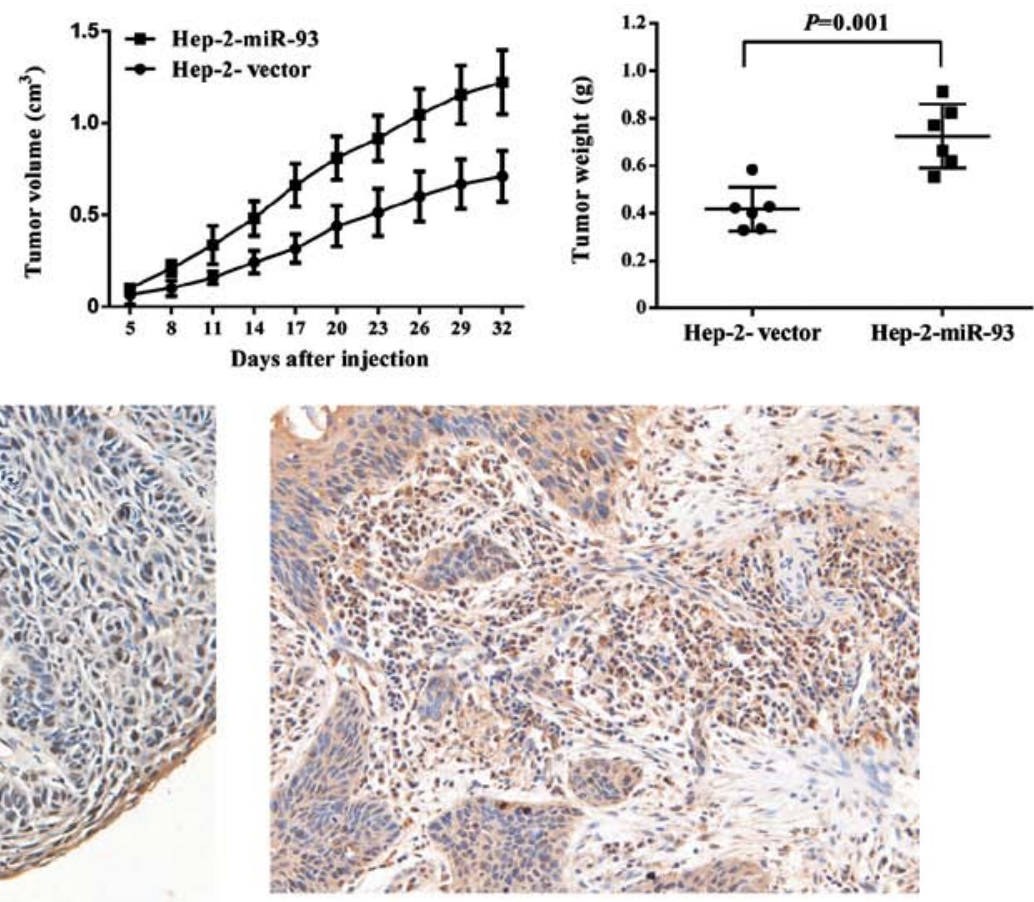

Hep-2-miR-93

Figure 5. miR-93 overexpression promotes tumor growth in mouse tumor xenografts. (A) Nude mice injected with Hep-2 cells stably overexpressing miR-93 or negative control. Tumors in each group were taken at the end point of the experiment. The tumor growth curve was shown and the weights of each group at the end of experiment were measured. (B) Tumor levels of Ki-67 protein of each group were measured by immunohistochemical staining. The Lenti-miR-93 group had a higher level of Ki-67.

investigate whether miR-93 could also promote cell invasion and metastasis in LSCC. Transwell assays without or with Matrigel demonstrated that the cells transfected with miR-93 displayed higher levels of migration and invasion than the mock-transfected cells (Fig. 3). In contrast, the silencing of endogenous miR-93 in Hep-2 cells decreased cell migration 
A

Site 1:

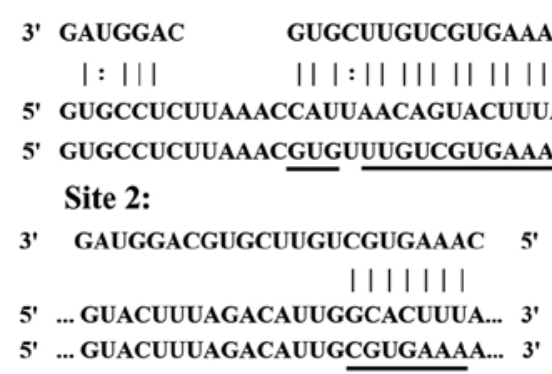

C

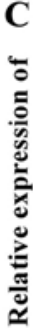

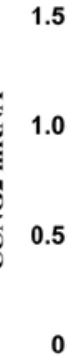

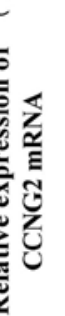

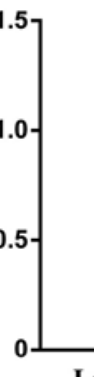

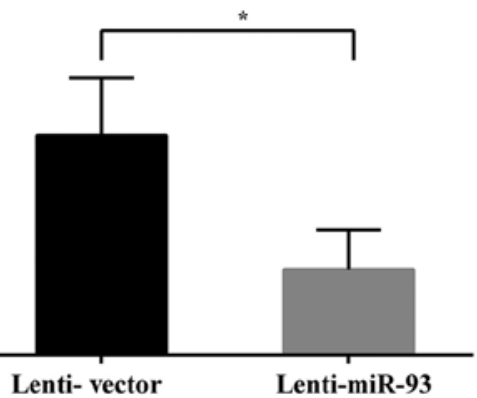

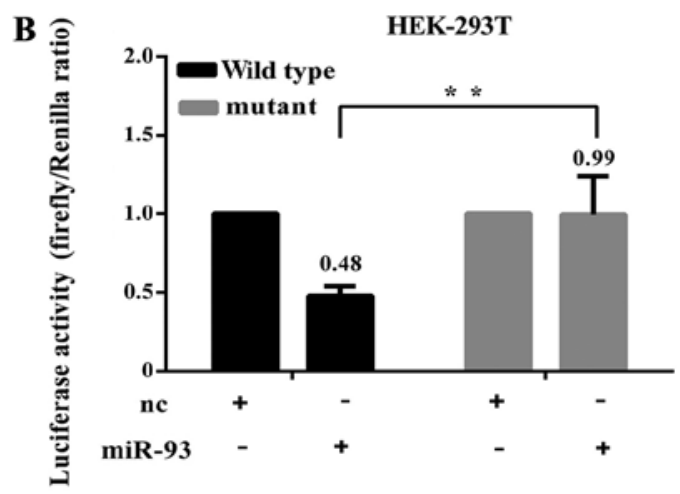

D
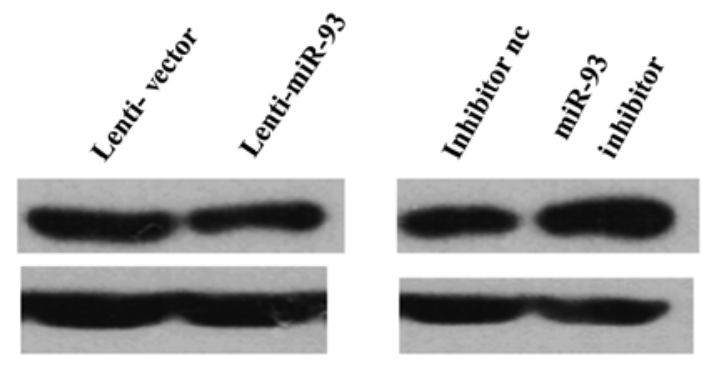

Figure 6. miR-93 negatively regulates CCNG2 by binding to the CCNG2 $3^{\prime} \mathrm{UTR}$. (A) Two putative miR-93-binding sites in CCNG2 3'UTR and the 2 corresponding mutant binding sites (underlined) are shown. (B) The relative luciferase activity of luciferase reports with wild-type or mutant CCNG2 3'UTRs was determined in HEK 293T cells, which were co-transfected with the miR-93 mimics or negative control. Statistical significance exists between the wild-type group and the mutant group with miR-93 transfection. Representative experiments are shown with the mean \pm SD. (C) The mRNA levels of CCNG2 were determined by qRT-PCR in stably miR-93 expressing cell line Hep-2. $\beta$-actin served as an internal control. (D) The protein levels of CCNG2 were determined by western blot assays in Hep-2 cells infected with miR-93 or mock vector and Hep-2 cells transfected with the miR-93 inhibitor or negative control.

and invasion (Fig. 3). Notably, the incubation times for the migration and invasion assays were 18 and $24 \mathrm{~h}$, respectively, and at those time-points, the cell growth was not significantly affected by miR-93. The migrated and invasive cells were stained and counted in six randomly selected fields under a light microscope. In contrast, the mRNA level of MMP-9 was upregulated in the Lenti-miR-93 cells, the fold change was 22.9 compared with Lenti-Vector cells. Taken together, these findings indicated that miR-93 promotes cancer cell migration and invasion in vitro.

miR-93 inhibits LSCC cell apoptosis in vitro and promotes cell cycle progression of LSCC cells. Given that miR-93 may function as an oncogene in LSCC, we considered whether miR-93 might have an important role in LSCC cell apoptosis. The flow cytometry assay demonstrated that the apoptosis rates of Hep-2 cells transfected with miR-93 mimics were significantly decreased compared with those of negative control miRNA-transfected cells $(\mathrm{P}<0.01)$. When transfected with miR-93 inhibitor, the apoptosis rates increased $(\mathrm{P}<0.01)$ (Fig. 4A). These results indicated that miR-93 inhibited the cellular apoptosis of laryngeal cancer cells.

To study whether miR-93 promoted the proliferation of LSCC carcinoma cells by affecting their cell cycle, cell cycle analysis was performed. Cell cycle analysis showed that after transfection with miR-93 mimics, the cell cycle was arrested at the $\mathrm{S}$ phase compared with cells transfected with mimics negative control in Hep- 2 cells. When transfection was carried out with miR-93 inhibitor, the cell cycle was arrested at G1 phase compared with cells transfected with inhibitor negative control in Hep-2 cells (Fig. 4B). These results suggested that miR-93 promoted the progression through the G1-S phase of the cell cycle.

miR-93 overexpression promotes tumor growth in laryngeal xenografts. To further characterize the oncogenic function of miR-93 in vivo, we generated subcutaneous xenografts using Hep-2 cells stably transfected with either miR-93 or a control construct. As shown in Fig. 5A, we found tumors in the mice injected with the Lenti-miR-93 cells, and significantly increased tumor growth in the nude mice compared to the Lenti-Vector cells. Fig. 5B shows that miR-93 overexpression increased expression of Ki-67 protein, a marker of proliferation, in tumor samples. Collectively, these findings demonstrated that miR-93 promotes LSCC cancer cell proliferation in vivo.

miR-93 represses $C C N G 2$ expression by directly targeting its $3^{\prime} U T R$. We investigated the target of miR-93 in mediating the observed effects focusing on tumor suppressors. The CCNG2 was identified as a potential target of miR-93. The 3'UTR of CCNG2 harbored two typical target sequences for miR-93 at nucleotides 186-214 and 222-229 (Fig. 6A). To obtain direct evidence that the 3'UTR of CCNG2 was a target of miR-93, we generated luciferase expression constructs harboring fragments of the CCNG2 3'UTR containing the miR-93 target sites, which produced the wild-type constructs CCNG2-186 and CCNG2-222. Two mutant constructs CCNG2-186-mutant and CCNG2-222-mutant were also generated (Fig. 6A). As 
A
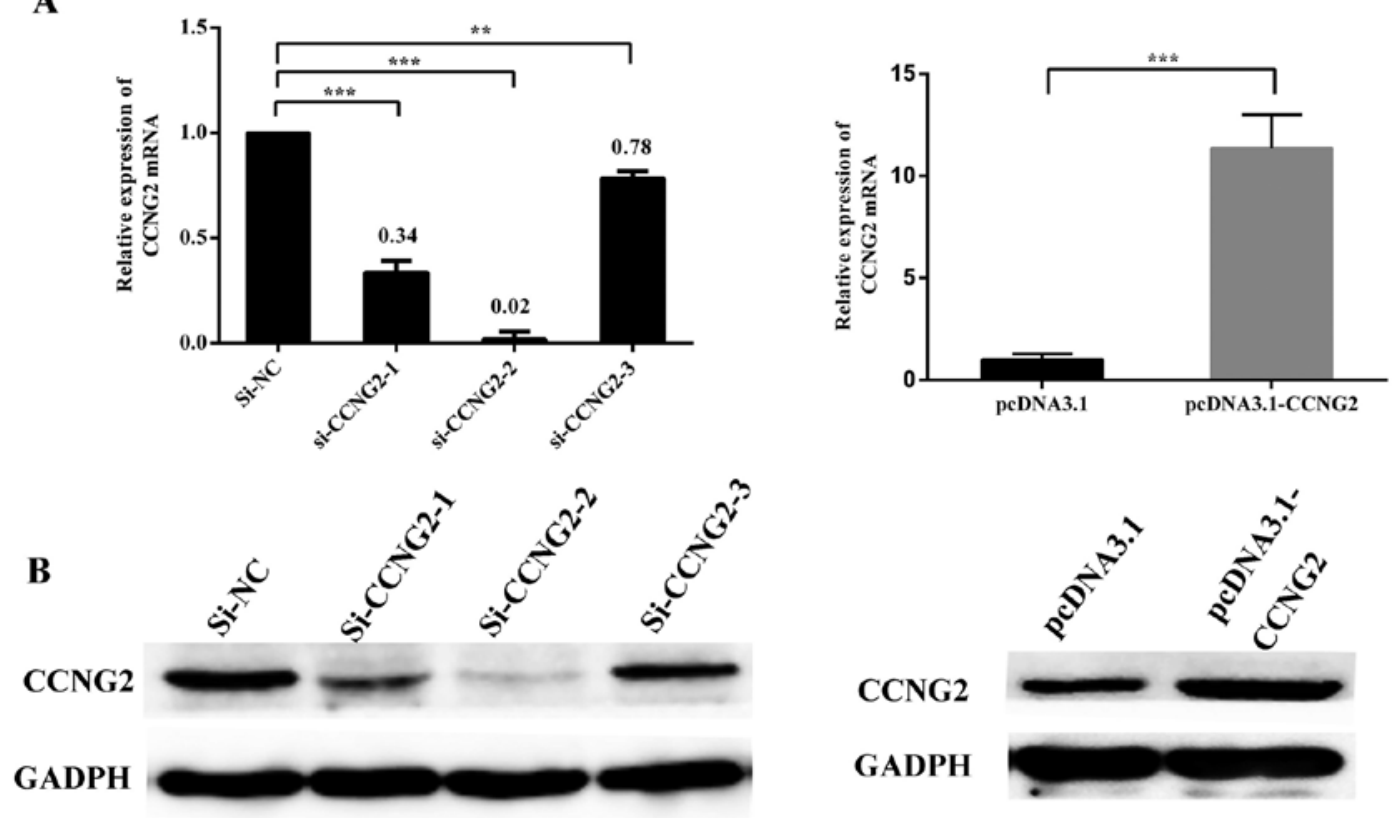

C
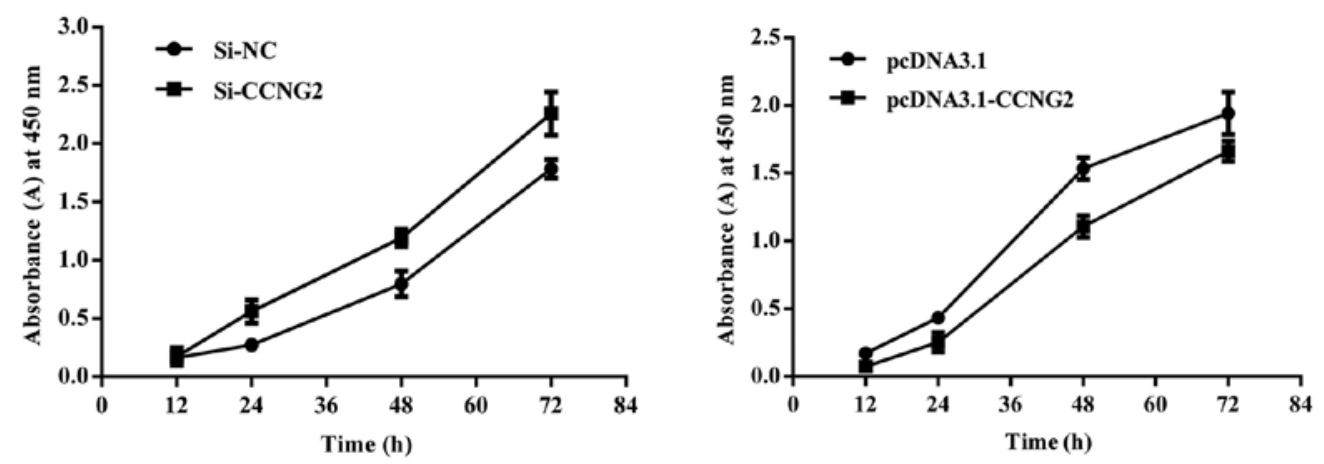

Figure 7. CCNG2 acts as a tumor suppressor in laryngeal carcinoma. (A) qRT-PCR of CCNG2 expression in Hep-2 cells transfected with siRNAs targeting CCNG2 or a negative control and Hep-2 cells transfected with a plasmid expressing CCNG2 or a negative control. (B) Western blot analysis of Hep-2 cells for CCNG2 protein expression after downregulation and upregulation of CCNG2, expression level of CCNG2 was normalized to GADPH. (C) Cell proliferation assay was carried out after transfection. Knockdown of CCNG2 promoted the proliferation of Hep-2 cells while overexpression of CCNG2 inhibited proliferation.

expected, expression of CCNG2-186 and CCNG2-222 showed that luciferase activities were significantly repressed when the constructs were cotransfected with miR-93 mimics. Mutations of the miR-93 target sites abolished the effects of miR-93 (Fig. 6B). The mRNA levels of CCNG2 were determined by qRT-PCR in Hep-2 cells stably expressing miR-93 or the negative control (Fig. 6C). In addition, western blot analyses showed that overexpression of miR-93 could lead to decreased CCNG2 protein level in Hep-2 cells. In contrast, miR-93 inhibitor increased the protein level of CCNG2 (Fig. 6D). Collectively, these results indicated that miR-93 could regulate CCNG2 expression by directly binding to its 3'UTR.

CCNG2 acts as a tumor suppressor in laryngeal carcinoma. To confirm that CCNG2 played an important role in mediating the miR-93 effects in carcinogenesis, we investigated the role of CCNG2 overexpression and knockdown in cultured Hep-2 cells. An expression plasmid vector pcDNA3.1 was used for overexpression, three siRNAs targeting CCNG2 were maintained in serum-free medium. Western blot assays showed that
CCNG2 protein levels were significantly increased by transfection of a CCNG2 expression construct, and were reduced by transfection of specific siRNA against CCNG2. As shown in Fig. 7A and B, since all siRNAs appeared to have functioned efficiently, one of them, the siRNA-2, was used for further analysis. CCNG2 overexpression significantly reduced cell proliferation as measured by CCK8, and after transfection with CCNG2-siRNA, cell proliferation of Hep-2 cells had increased markedly as compared with cells transfected with the control oliogo (Fig. 7C). To examine if ectopic expression of CCNG2 altered the cell cycle profile in Hep-2 cells, we determined the cell cycle positions. Compared with control transfectants, CCNG2-overexpression cells showed a decrease in $\mathrm{S}$ phase and an increase in G1 phase. Knockdown of CCNG2 expression leads to an increase in $\mathrm{S}$ phase, the cell cycle was arrested at $\mathrm{S}$ phase (Fig. 8A). To examine if ectopic expression of CCNG2 induced apoptosis, we measured percentage of apoptotic cell population by Annexin V-FITC/PI analysis. Consitent with the observation of the proliferation and cell cycle, we observed CCNG2-overexpression significantly induced apoptosis 


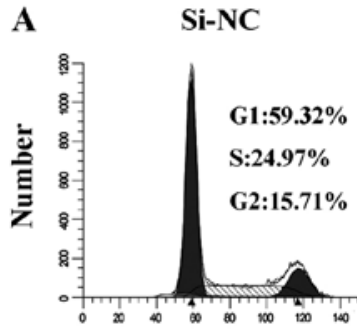

FL 3 Area-PI

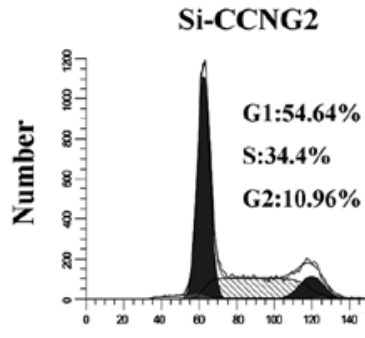

FL 3 Area-PI

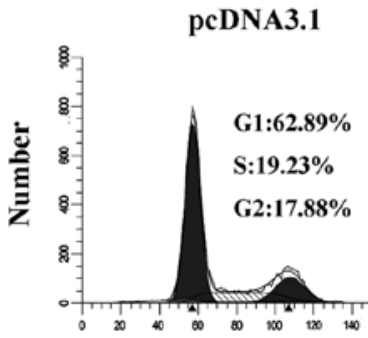

FL 3 Area-PI

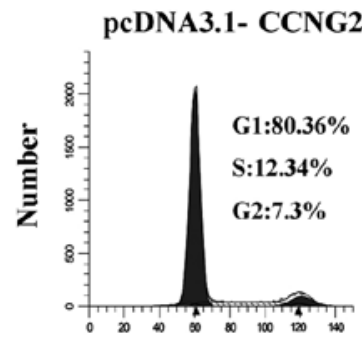

FL 3 Area-PI
B

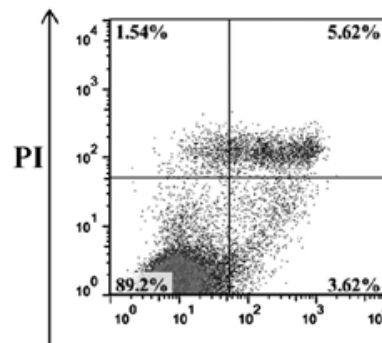

Si-CCNG2

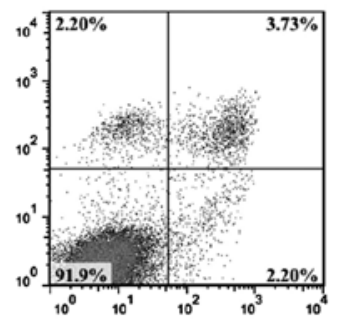

pcDNA3.1

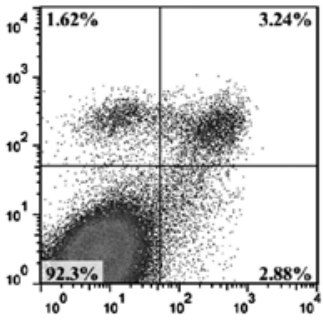

peDNA3.1-CCNG2

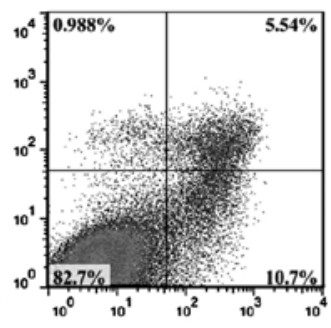

AnnexinV-FITC

Figure 8. CCNG2 acts as a tumor suppressor in laryngeal carcinoma and plays an important role in cell cycle and cells apoptosis. (A) Knockdown of CCNG2 in Hep-2 cells induced cell cycle arrest at S phase. Higher expression of CCNG2 in Hep-2 cells induced cell cycle arrested at the G1 phase. (B) Knockdown of CCNG2 reduced cell apoptosis rates of Hep-2 cells. Higher expression of CCNG2 in Hep-2 cells increased cell apoptosis rates.

compared with null plasmid transfection $(\mathrm{P}<0.01)$ (Fig. 8B). These results indicate that $\mathrm{CCNG} 2$ inhibits growth of LSCC cells by inducing cell arrest at the G1/S checkpoint of the cell cycle, the downregulation of CCNG2 promotes the proliferation and cell cycle progress of LSCC cells. These data support the growth inhibitory function of $\mathrm{CCNG} 2, \mathrm{CCNG} 2$ acted as a tumor suppressor in laryngeal carcinoma.

Confirmation of the targeting effects by rescue experiments. Rescue experiments were performed to further confirm that miR-93 promoted cell proliferation and migration by targeting CCNG2. Hep-2 cells stably transfected with miR-93 were transfected with pcDNA3.1-CCNG2 plasmid or a control plasmid. Western blot analysis was used as confirmation of the expression for the protein gel blot probed with anti-CCNG2 antibody (Fig. 9A). Cell proliferation assay showed that reintroduction of CCNG2 into the miR93-expressing cells reversed the effect of miR-93 on cell proliferation (Fig. 9B) and failed to promote the cell cycle process (Fig. 9D). Decreased migration was found in cells transfected with CCNG2 (Fig. 9C). The flow cytometry data showed that overexpression of CCNG2 in miR-93 cells can attenuate the anti-apoptotic effect of miR-93 (Fig. 9E). Thus, re-expression of CCNG2 was sufficient to cause cell death and decrease both cell proliferation and migration. This suggested that the effects of miR-93 on enhanced proliferation and metastasis were at least partly taking place through repression of CCNG2 expression.

\section{Discussion}

The role of miR-93 in cancer is controversial because reports have indicated that it can be tumor suppressive or tumor promoting, depending on context. miR-93 is upregulated in a subset of human tumors and leads to deregulation of important cancer-related genes (23-27) whereas, in some types of cancer, miR-93 represses proliferation paradoxically (28). Despite increasing evidence pointing to a role for miR-93 as a tumor oncomiR, the tumor promotion effect of miR-93 has not been fully elucidated in LSCC. In the present study, we provide the first integrated investigation of the function and probable underlying mechanisms of miR-93 and CCNG2 in LSCC at both cellular and clinical levels.

Previous data showed that as a key negative regulator of cell cycle proliferation, apoptosis, cell death signaling, and even carcinogenesis, suggesting that the decreased expression levels of CCNG2 significantly correlates with tumor progression and unfavorable prognosis, such as studies in colorectal carcinoma, kidney, esophageal, oral cancer, gastric carcinoma, prostate cancer and papillary carcinoma of the thyroid (29-35). In the present study, we found that $\mathrm{CCNG} 2$ expression was markedly decreased in the LSCC tissues compared with the adjacent normal tissues and lower expression of CCNG2 tended to have more advanced clinical stage. The expression of CCNG2 in laryngeal carcinoma tissues without lymph node metastasis or with high differentiation presented to be higher than laryngeal carcinoma tissues with lymph node metastasis or with poor differentiation, suggesting that downregulation of CCNG2 expression in LSCC is associated with malignant degree and development tendency. We further demonstrate that CCNG2 functions as a potent repressor of cell proliferation and invasion at cellular levels.

The downregulation of CCNG2 expresion in cancer cells may be caused by multiple mechanisms, as we show here, including through miRNA regulation. We verified that miR-93 regulates CCNG2 expression by directly targeting the 
A

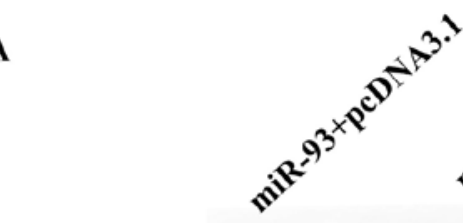

CCNG2

\section{GADPH}

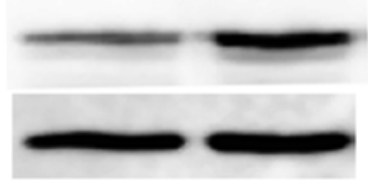

B

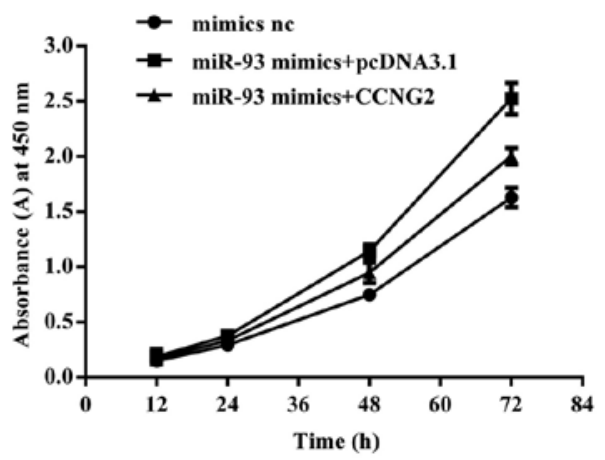

C

miR-93+pcDNA3.1

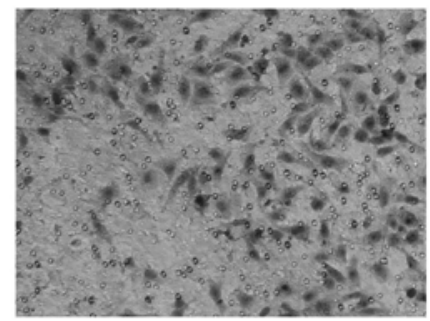

D

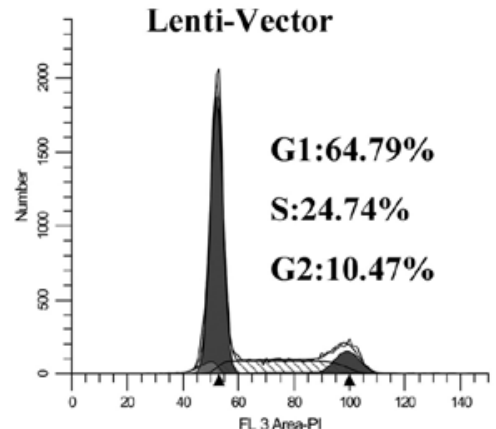

E

Lenti-Vector
miR-93+CCNG2
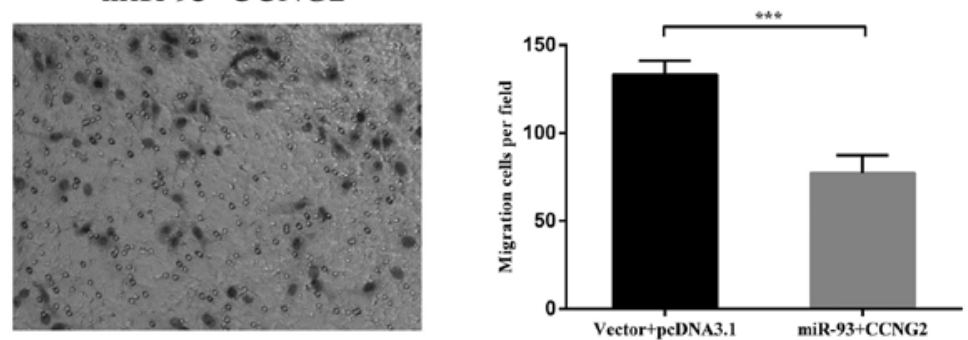

miR-93+CCNG2

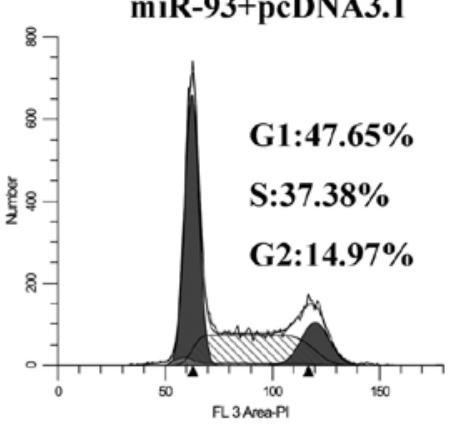

miR-93+pcDNA3.1

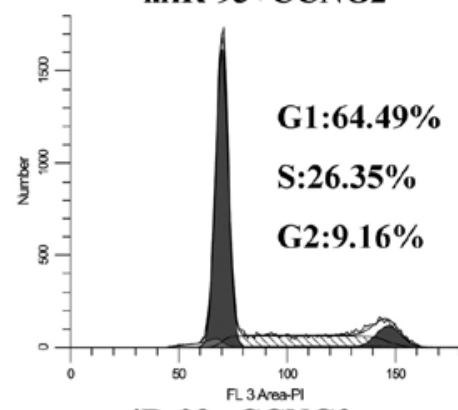

miR-93+ CCNG2
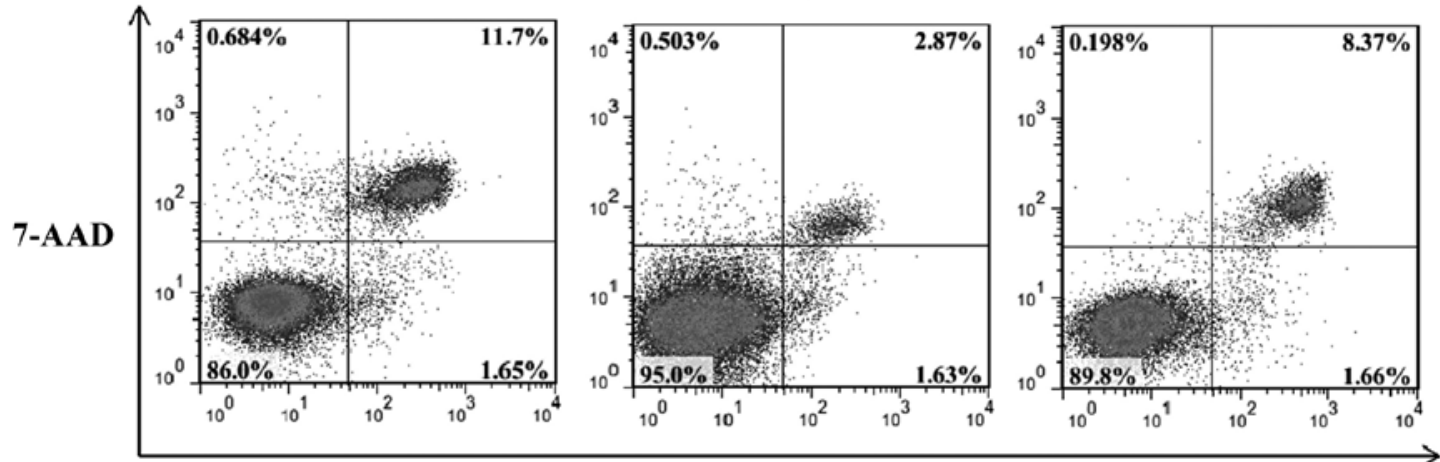

Annexin V-APC

Figure 9. CCNG2 mediates the effects of miR-93. (A) Cell lysates prepared from Lenti-miR-93 cells transfected with CCNG2 plasmid or a control were subjected to western blot analysis for re-expression of CCNG2. As expected, transfection with CCNG2 increased its expression. (B) Ectopic expression of CCNG2 in miR-93 cells reversed miR-93 effect on cell proliferation. (C) Ectopic expression of CCNG2 in the miR-93 cells reversed the miR-93 effect on cell migration. (D) Re-expression of CCNG2 in Hep-2 cells reversed the effect of miR-93 on the cell cycle. (E) The flow cytometry data showed that overexpression of CCNG2 in miR-93 cells can attenuate the anti-apoptotic effect of miR-93.

3'UTR of CCNG2 mRNA. There was a significant negative correlation between CCNG2 expression and miR-93 levels in laryngeal cancer specimens, confirming the role of miR-93 in regulating CCNG2 expression. After validation experiments, we found that the aberrant overexpression of miR-93 markedly attenuated CCNG2 expression at the mRNA and protein level in the Hep- 2 cells. Data of luciferase reporter assay showed that miR-93 could bind to the wild-type target sequence but not the mutant target sequence. Therefore, to a certain extent, this result supports our hypothesis that CCNG2 is a direct target gene of miR-93 in Hep-2 cells, miR-93 has the primary role in determining CCNG2 expression in laryngeal cancer. Silencing of CCNG2 expression by small interfering RNAs indicated that $\mathrm{CCNG} 2$ plays crucial roles in cell proliferation. In addition, rescue experiments demonstrated that $\mathrm{CCNG} 2$ re-expression can only partly reverse the function of miR-93, 
which may suggest that CCNG2 was not the only target of miR-93 in laryngeal carcinoma and thus there may be some other targets of miR-93.

It is well recognized that enhanced cell proliferation, cell cycle arrest, resistance to apoptosis and the migration state of LSCC cells plays key roles in the progression of LSCC (36-39). Another important finding of our study is that miR-93 acted as an oncomiR affecting the proliferation, cell cycle progress, apoptosis and migration state of LSCC cells. Firstly, we found that elevated miR-93 levels promoted the growth of Hep-2 cells in vitro and in vivo which was associated with the inhibition of apoptosis and induction of cell cycle arrest. In vivo tumor formation assays, the Lenti-miR-93 group displayed stronger Ki-67 immunoreactivity, an indication of extensive cell proliferation, than Lenti-Vector group. Secondly, miR-93 appeared to play roles in migraton and invasion of Hep-2 cells, cellular migration and invasion was enhanced following ectopic expression of miR-93 in the Hep-2 cells. To further confirm whether silencing of miR-93 inhibits proliferation, clonability, migratory and invasion potential, we silenced miR-93 in Hep-2 cells using miR-93 inhibitor and all the assays discussed above were carried out. On the contrary, knocking down the expression of miR-93 performed the reverse function. All of the above indicated an oncogenic role of miR-93 in LSCC carcinogenesis.

The dysregulation of cell cycle control and apoptosis state is emerging as a central theme of carcinogenesis. Cyclin G2 is an unconventional cyclin highly expressed in cells undergoing apoptosis, its expressiom is upregulated as cells undergo cell cycle arrest or apoptosis in response to inhibitory stimuli independent of P53 $(40,41)$. In lymphocytes, cyclin G2 expression oscillating in late S/early G2 phase of cell cycle (42). Correspondingly, CCNG2 has a potential pro-apoptotic role; however, the underlying mechanisms remain unclear. However, it has been shown that there are two different mechanisms associated with cell apoptosis: the extrinsic receptor-mediated pathway and the intrinsic mitochondrial-dependent pathway (43). Our results revealed that the overexpression of miR-93 markedly downregulated CCNG2 expression, and finally induced cell cycle $\mathrm{S}$ phase blockage in the Hep- 2 cells. Our results also indicated that the anti-apoptotic effect of miR-93 in Hep-2 cells is partially dependent on CCNG2-mediated mitochondrial apoptotic signaling.

To initiate the metastatic process, cancer cells must first penetrate the epithelial basement membrane. The degradation of extracellular matrix (ECM) is associated with cell invasion. Among matrix metalloproteinases (MMPs), MMP-9 digest type IV collagen, a major compotent of basement membrane. Thus, MMP-9 plays a central role in cancer cell invasion and is frequently upregulated in cancer cells $(44,45)$. In the present study, the miR-93 Hep-2 cells were able to penetrate through the Matrigel as compared with the control cells. It appeared that the miR-93 Hep-2 cells were able to digest the matrix molecules, which facilitated cell invasion and led to metastasis. The mRNA level of MMP-9 were upregulated in the miR-93-transfected cells, which significantly stimulated the migratory capability of Hep-2 cells, supporting that miR-93 might promote cell migration through the CCNG2-MMP-9 pathway, partly due to its effect on MMP-9. The specific molecular mechanisms of this pathway remain to explore further.

In summary, miR-93, as an oncomiR, promotes proliferation, inhibits apoptosis, induces cell cycle arrest and promotes the migration and invasion of LSCC cells by targeting CCNG2. Intriguingly, relatively high levels of CCNG2 predicted a better outcome in LSCC patients and function as a prognostic factor in LSCC. We also found that miR-93 and CCNG2 expression were inversely associated in LSCC samples. The associations of CCNG2 levels with clinical stages, lymph node metastasis, and histological grade further demonstrate the relevance of the miR-93/CCNG2 pathway in LSCC tumorigenesis. Given the increasing appreciation of miRNAs in cancer therapeutics and diagnostics, this novel oncogenic pathway clearly warrants further investigation to determine whether new therapeutic tools and prognostic biomarkers for laryngeal cancer can be developed.

\section{Acknowledgements}

The present study was financially supported by the Key Project of Shanghai Science and Technology Committee (grant no. 12JC1402100).

\section{References}

1. Ferlay J, Shin HR, Bray F, et al: Estimates of worldwide burden of cancer in 2008: GLOBOCAN 2008. Int J Cancer 127: 2893-2917, 2010.

2. Marioni G, Marchese-Ragona R, Cartei G, et al: Current opinion in diagnosis and treatment of laryngeal carcinoma. Cancer Treat Rev 32: 504-515, 2006.

3. Talamini R, Bosetti C, La Vecchia C, et al: Combined effect of tobacco and alcohol on laryngeal cancer risk: a case-control study. Cancer Causes Control 13: 957-964, 2002.

4. Menach P, Oburra HO and Patel A: Cigarette smoking and alcohol ingestion as risk factors for laryngeal squamous cell carcinoma at Kenyatta National Hospital, Kenya. Clin Med Insights Ear Nose Throat 5: 17-24, 2012.

5. Mohamadian Roshan N, Jafarian A, Ayatollahi H, et al: Correlation of laryngeal squamous cell carcinoma and infections with either HHV-8 or HPV-16/18. Pathol Res Pract 210: 205-209, 2014.

6. Hoffman HT, Porter K, Karnell LH, et al: Laryngeal cancer in the United States: changes in demographics, patterns of care, and survival. Laryngoscope 116 (Suppl 111): 1-13, 2006.

7. Zhang B, Pan X, Cobb GP, et al: microRNAs as oncogenes and tumor suppressors. Dev Biol 302: 1-12, 2007.

8. Shah MY and Calin GA: MicroRNAs as therapeutic targets in human cancers. Wiley Interdiscip Rev RNA 5: 537-548, 2014.

9. Kong YW, Ferland-McCollough D, Jackson TJ, et al: microRNAs in cancer management. Lancet Oncol 13: e249-258, 2012.

10. Calin GA, Sevignani C, Dumitru CD, et al: Human microRNA genes are frequently located at fragile sites and genomic regions involved in cancers. Proc Natl Acad Sci USA 101: 2999-3004, 2004.

11. Yeung ML, Yasunaga J, Bennasser Y, et al: Roles for microRNAs, miR-93 and miR-130b, and tumor protein 53-induced nuclear protein 1 tumor suppressor in cell growth dysregulation by human T-cell lymphotrophic virus 1. Cancer Res 68: 8976-8985, 2008.

12. Du L, Schageman JJ, Subauste MC, et al: miR-93, miR-98, and miR-197 regulate expression of tumor suppressor gene FUS1. Mol Cancer Res 7: 1234-1243, 2009.

13. Singh B, Ronghe AM, Chatterjee A, et al: MicroRNA-93 regulates NRF2 expression and is associated with breast carcinogenesis. Carcinogenesis 34: 1165-1172, 2013.

14. Fang L, Deng Z, Shatseva T, et al: MicroRNA miR-93 promotes tumor growth and angiogenesis by targeting integrin- $\beta 8$. Oncogene 30: 806-821, 2011. 
15. Fang L, Du WW, Yang W, et al: MiR-93 enhances angiogenesis and metastasis by targeting LATS2. Cell Cycle 11: 4352-4365, 2012.

16. Fu X, Tian J, Zhang L, et al: Involvement of microRNA-93, a new regulator of PTEN/Akt signaling pathway, in regulation of chemotherapeutic drug cisplatin chemosensitivity in ovarian cancer cells. FEBS Lett 586: 1279-1286, 2012.

17. Liu S, Patel SH, Ginestier C, et al: MicroRNA93 regulates proliferation and differentiation of normal and malignant breast stem cells. PLoS Genet 8: e1002751, 2012.

18. Bennin DA, Don AS, Brake T, et al: Cyclin G2 associates with protein phosphatase $2 \mathrm{~A}$ catalytic and regulatory $\mathrm{B}^{\prime}$ subunits in active complexes and induces nuclear aberrations and a G1/S phase cell cycle arrest. J Biol Chem 277: 27449-27467, 2002

19. Burgering BM and Kops GJ: Cell cycle and death control: long live Forkheads. Trends Biochem Sci 27: 352-360, 2002.

20. Martínez-Gac L, Marqués M, García Z, et al: Control of cyclin G2 mRNA expression by forkhead transcription factors: novel mechanism for cell cycle control by phosphoinositide 3-kinase and forkhead. Mol Cell Biol 24: 2181-2189, 2004.

21. Arachchige Don AS, Dallapiazza RF, Bennin DA, et al: Cyclin G2 is a centrosome-associated nucleocytoplasmic shuttling protein that influences microtubule stability and induces a p53-dependent cell cycle arrest. Exp Cell Res 312: 4181-4204, 2006.

22. Cao P, Zhou L, Zhang J, et al: Comprehensive expression profiling of microRNAs in laryngeal squamous cell carcinoma. Head Neck 35: 720-728, 2013.

23. Wang L, Wang Q, Li HL, et al: Expression of MiR200a, miR93, metastasis-related gene RECK and MMP2/MMP9 in human cervical carcinoma--relationship with prognosis. Asian Pac J Cancer Prev 14: 2113-2118, 2013.

24. Kim BH, Hong SW, Kim A, et al: Prognostic implications for high expression of oncogenic microRNAs in advanced gastric carcinoma. J Surg Oncol 107: 505-510, 2013.

25. Xu D, He XX, Chang Y, et al: Downregulation of MiR-93 expression reduces cell proliferation and clonogenicity of HepG2 cells. Hepatogastroenterology 59: 2367-2373, 2012.

26. Chen L, Jiang M, Yuan W, et al: Prognostic value of miR-93 overexpression in resectable gastric adenocarcinomas. Acta Gastroenterol Belg 75: 22-27, 2012.

27. Montanini L, Lasagna L, Barili V, et al: MicroRNA cloning and sequencing in osteosarcoma cell lines: differential role of miR-93. Cell Oncol (Dordr) 35: 29-41, 2012.

28. Yu XF, Zou J, Bao ZJ, et al: miR-93 suppresses proliferation and colony formation of human colon cancer stem cells. World $\mathrm{J}$ Gastroenterol 17: 4711-4717, 2011.

29. Sun GG, Zhang J and Hu WN: CCNG2 expression is downregulated in colorectal carcinoma and its clinical significance. Tumour Biol 35: 3339-3346, 2014.

30. Cui DW, Sun GG and Cheng YJ: Change in expression of cyclin G2 in kidney cancer cell and its significance. Tumour Biol 35: 3177-3183, 2014.
31. Chen JQ, Liu CJ, Wen HX, et al: Changes in the expression of cyclin G2 in esophageal cancer cell and its significance. Tumour Biol 35: 3355-3362, 2014.

32. Kim Y, Shintani S, Kohno Y, et al: Cyclin G2 dysregulation in human oral cancer. Cancer Res 64: 8980-8986, 2004.

33. Sun GG, Hu WN, Cui DW, et al: Decreased expression of CCNG2 is significantly linked to the malignant transformation of gastric carcinoma. Tumour Biol 35: 2631-2639, 2014.

34. Cui DW, Cheng YJ, Jing SW, et al: Effect of cyclin G2 on proliferative ability of prostate cancer PC-3 cell. Tumour Biol 35: 3017-3024, 2014

35. Ito Y, Yoshida H, Uruno T, et al: Decreased expression of cyclin G2 is significantly linked to the malignant transformation of papillary carcinoma of the thyroid. Anticancer Res 23: 2335-2338, 2003.

36. Jiao J, Qin Z, Li S, et al: Potential role of Notch1 signaling pathway in laryngeal squamous cell carcinoma cell line Hep-2 involving proliferation inhibition, cell cycle arrest, cell apoptosis, and cell migration. Oncol Rep 22: 815-823, 2009.

37. Ren J, Zhu D, Liu M, et al: Downregulation of miR-21 modulates Ras expression to promote apoptosis and suppress invasion of Laryngeal squamous cell carcinoma. Eur J Cancer 46: 3409-3416, 2010.

38. Che XH, Chen H, Xu ZM, et al: 14-3-3epsilon contributes to tumour suppression in laryngeal carcinoma by affecting apoptosis and invasion. BMC Cancer 10: 306, 2010.

39. Tian L, Li M, Ge J, et al: MiR-203 is downregulated in laryngeal squamous cell carcinoma and can suppress proliferation and induce apoptosis of tumours. Tumour Biol 35: 5953-5963, 2014.

40. Horne MC, Goolsby GL, Donaldson KL, et al: Cyclin G1 and cyclin G2 comprise a new family of cyclins with contrasting tissue-specific and cell cycle-regulated expression. J Biol Chem 271: 6050-6061, 1996

41. Horne MC, Donaldson KL, Goolsby GL, et al: Cyclin G2 is up-regulated during growth inhibition and $\mathrm{B}$ cell antigen receptor-mediated cell cycle arrest. J Biol Chem 272: 12650-12661, 1997.

42. Bates S, Rowan S and Vousden KH: Characterisation of human cyclin G1 and G2: DNA damage inducible genes. Oncogene 13: 1103-1109, 1996.

43. Wang DH, Hu JR, Wang LY, et al: The apoptotic function analysis of p53, Apaf1, Caspase 3 and Caspase7 during the spermatogenesis of the Chinese fire-bellied newt Cynops orientalis. PLoS One 7: e39920, 2012.

44. Johansson N, Ahonen M and Kähäri VM: Matrix metalloproteinases in tumor invasion. Cell Mol Life Sci 57: 5-15, 2000.

45. Fink K and Boratyński J: The role of metalloproteinases in modification of extracellular matrix in invasive tumor growth, metastasis and angiogenesis. Postepy Hig Med Dosw (Online) 66: 609-628, 2012 (In Polish). 